\title{
Graphene-Based Nanomaterials for Bioimaging
}

\author{
Jing Lin, ${ }^{\mathrm{a}}$ Xiaoyuan Chen ${ }^{\mathrm{b}, *}$, Peng Huang ${ }^{\mathrm{a}, *}$ \\ ${ }^{a}$ Guangdong Key Laboratory for Biomedical Measurements and Ultrasound Imaging, \\ Department of Biomedical Engineering, School of Medicine, Shenzhen University, Shenzhen \\ 518060, China
}

${ }^{b}$ Laboratory of Molecular Imaging and Nanomedicine (LOMIN), National Institute of

Biomedical Imaging and Bioengineering (NIBIB), National Institutes of Health, Bethesda,

Maryland 20892, United States

* Address correspondence to shawn.chen@nih.gov or peng.huang@szu.edu.cn. 


\section{Abstract}

Graphene-based nanomaterials, due to their unique physicochemical properties, versatile surface functionalization, ultra-high surface area, and good biocompatibility, have attracted considerable interest in biomedical applications such as biosensors, drug delivery, bioimaging, theranostics, and so on. In this review, we will summarize the current advances in bioimaging of graphenebased nanomaterials, including graphene, graphene oxide $(\mathrm{GO})$, reduced graphene oxide ( $\mathrm{rGO}$ ), graphene quantum dots (GQDs), and their derivatives. There are two methods to synthesize graphene-based nanomaterials: in situ synthesis and binding method. We will highlight the molecular imaging modalities including optical imaging (fluorescence (FL), two-photon FL, and Raman imaging), PET/SPECT (positron emission tomography/single photon emission computed tomography), MRI (magnetic resonance imaging), PAI (photoacoustic imaging), CT (computed tomography), and multimodal imaging. In the end, we will elaborate on the prospects and challenges of their future bioimaging applications.

Keywords: Graphene, graphene oxide, reduced graphene oxide, graphene quantum dots, photoacoustic imaging, PET/SPECT, MRI, bioimaging 


\section{Introduction}

Graphene, an atomically thick sheet of two-dimensional (2D) honeycomb monolayer $s p^{2}$ hybridized carbon atoms, is the basic building block for all other dimensionalities of carbon nanomaterials, such as fullerene, carbon nanotubes, and carbon nanohorns (1-3). Since its isolation of in 2004, graphene and its derivatives have gained considerable attention in chemistry, materials, physics and biomedical communities (4). Afterwards, the new members of graphene family, such as graphene oxide $(\mathrm{GO})(5,6)$, reduced graphene oxide $(\mathrm{rGO})(7,8)$, graphene quantum dots (GQDs) (9-11), and their derivatives were extensively explored in biosensors, drug delivery, bioimaging, theranostics, and so on (12-16).

Graphene with aromatic structure has many carbon-carbon bonds in the plane, which provide free $\pi$ electrons and reactive sites for surface reactions (3). GO, graphene's water-soluble derivative, is a highly oxidized form of chemically modified graphene, which consists of single atom thick layer of graphene sheets with hydroxyl (-OH) and epoxide (-O-) functional groups on the two accessible sides, and carboxylic acid $(-\mathrm{COOH})$ groups at the edges $(17,18)$. The unmodified areas of graphene in GO containing free $\pi$ electrons are hydrophobic and capable of drug loading and non-covalent surface modification by $\pi-\pi$ stacking and hydrophobic interactions $(19,20)$. The epoxide, hydroxyl, and carboxylic acid groups of GO are uncharged but polar, allowing weak interactions, hydrogen bonding and other surface reactions (21). By 
reduction treatment of GO, the oxygen content, surface charge, and hydrophilicity of GO are decreased, and then rGO is produced with restored electrical conductivity and enhanced optical absorbance $(8,22)$. GQDs are two-dimensional graphene fragments sized in 10-60 nm, usually not single-layer but multi-layers ( 10 layers of rGO) (23).

The biomedical applications of graphene and its derivatives are extensively investigated for diagnostics, drug delivery, near-infrared (NIR) light induced photothermal therapy and bioimaging (12). Among them, bioimaging plays critical roles in both research and clinical practice, which allows the observation and study of biological processes from the cellular and subcellular levels to small animals (24). By exploiting specific molecular probes or contrast agents, this powerful technique can detect and characterize early stage disease and provide a rapid method to monitor the treatment response (24). Due to their versatile surface functionalization and ultra-high surface area, graphene and its derivatives can be easily functionalized by small molecular dyes, polymers, nanoparticles, drugs or biomolecules to obtain graphene-based nanomaterials for different bioimaging applications (13). Herein, we focus on applying graphene-based nanomaterials for different molecular imaging modalities, including optical imaging (FL, two-photon FL, and Raman imaging), PET/SPECT (positron emission tomography/single photon emission computed tomography), MRI (magnetic resonance imaging), PAI (photoacoustic imaging), CT (computed tomography), and multimodal imaging (Fig. 1). 
Each imaging modality will be discussed in details in the subsequent sections. In the end, we will briefly discuss the prospects and challenges on their future bioimaging applications.

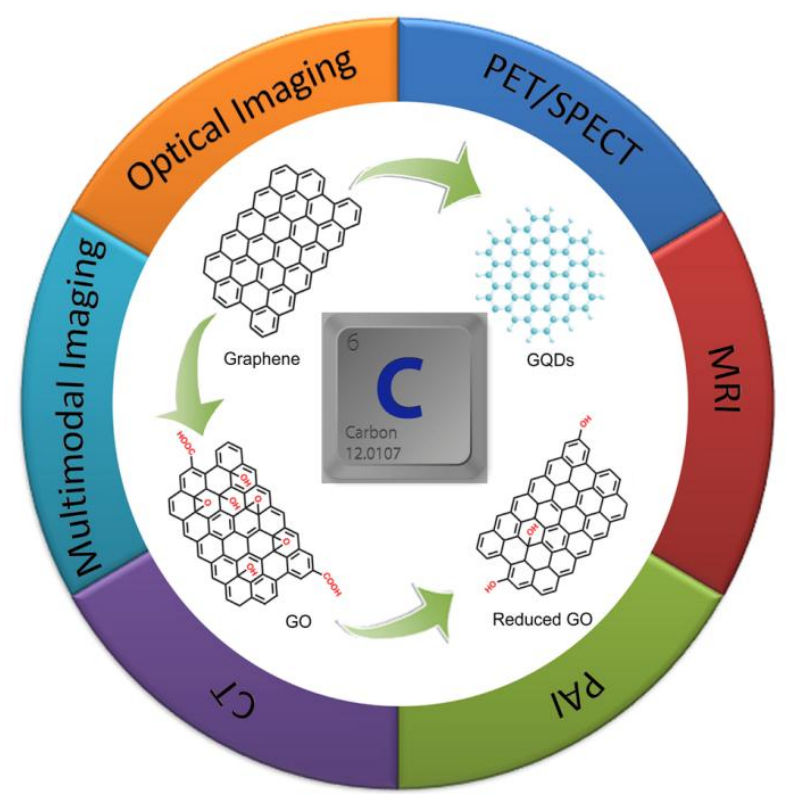

Fig. 1. Graphene-based nanomaterials, such as graphene, graphene oxide (GO), reduced graphene oxide (Reduced GO), and graphene quantum dots (GQDs) for molecular imaging, including optical imaging (fluorescence (FL), two-photon FL and Raman imaging), PET/SPECT, MRI (magnetic resonance imaging), PAI (photoacoustic imaging), CT (computed tomography), and multimodal imaging.

\section{Synthesis of graphene-based nanomaterials}

\subsection{Synthesis of graphene, GO, reduced GO, and GQDs}


Graphene can be synthesized by either bottom-up or top-down strategy (25). The bottomup strategy mainly involves chemical vapor deposition (CVD), organic synthesis, and solvothermal synthesis (13). The top-down strategy mainly involves mechanical, physical and chemical exfoliation methods (26). GOs are typically produced by the Hummer's method through the oxidative exfoliation of graphite using $\mathrm{KMnO}_{4} / \mathrm{H}_{2} \mathrm{SO}_{4}$ (27). Reduced $\mathrm{GO}$ can be obtained by treating GO with reducing agents, such as hydrazine, hydrazine hydrate, L-ascorbic acid, and so on (18). GQDs are usually prepared by thermal oxidation of GOs or other carbon precursors $(28,29)$.

\subsection{In situ growth method}

GO has been employed as a template to direct the synthesis of inorganic nanomaterials, such as iron oxide (30), gold nanoparticles (31), silver nanoparticles (32), and so on. Some functional groups of GO, such as carboxylic acid and hydroxyl groups, can serve as the binding sites with metal ions, and form nuclei surrounding the interaction sites between metal ions and GO, and followed by the growth of nanomaterials around the nucleus (32). For instance, GO/iron oxide composites can be synthesized by the following methods: $\mathrm{Fe}^{3+} / \mathrm{Fe}^{2+}$ ions in the proper molar ratio (2:1) were mixed with GO, and then were precipitated by alkaline solutions, such as 
ammonia solution $\left(\mathrm{NH}_{3} \cdot \mathrm{H}_{2} \mathrm{O}\right)$, and sodium hydroxide $(\mathrm{NaOH})(33,34)$. Besides the coprecipitation strategy of $\mathrm{Fe}^{3+}$ and $\mathrm{Fe}^{2+}$ ions under alkaline condition, hydrothermal reaction was also employed to prepare GO/iron oxide composites by using iron chloride hexahydrate as the iron source $(30,35)$. The as-prepared GO/iron oxide composites can be used as MR contrast agents for MRI. GO/gold composites or GO/silver composites can be prepared by incubating metal ions $\left(\mathrm{AuCl}_{4}^{-}, \mathrm{Ag}^{+}\right.$, etc. $)$with $\mathrm{GO}$, and then followed by the reduction of these metal ions (32). With different reducing agents, the size, shape, and morphology of metal nanoparticles can be controlled. The GO/metal composites can be used for X-ray CT imaging. These in situ synthetic routes are simple and effective for cost-effective large-scale production. Additionally, the $\mathrm{GO} /$ metal composites prepared by this method is rather stable, due to the strong covalent binding of $\mathrm{GO}$ and inorganic nanomaterials.

There are several challenges for the in situ growth method: i) How to obtain GO with uniform size and narrow distribution? Current synthesis methods are usually based on the top down strategy. The bottom up strategy may have potential to make very uniform GO. ii) How to control the functional groups on GO? These groups directly determine the number, density, and location of the nuclei of inorganic nanomaterials on GO, which ultimately influence the quality and properties of graphene-based nanomaterials. 


\subsection{Binding method}

Although the in situ synthetic strategies can directly control the growth of inorganic nanomaterials on the surface of $\mathrm{GO} / \mathrm{rGO}$, it often does not provide a good control over the particle size and morphology (36). To precisely control the particle size and morphology, there is an alternative strategy using a two-step binding method: i) prepare the inorganic nanomaterials by their preferred synthetic route. ii) deposit these as-prepared nanomaterials onto GO sheets through physical adsorption, van der Waals forces, electrostatic binding, or charge transfer interactions (37). This strategy doesn't affect the graphene's natural structure and appears to be more versatile (36). Meanwhile, this method offers good control over the size, shape, and functionality of nanomaterials. For example, Chen et al. incorporated 11-mercaptoundecanoic acid (MUA) capped-CdSe/ZnS quantum dots (QDs) with amphiphilic poly(L-lysine) modified rGO through the electrostatic interactions (38). Bovine serum albumin (BSA) capped-QDs can also be grafted onto polyethylenimine (PEI) adsorbed rGO (39). In another case, the dodecanethiol-CTAB-capped gold nanoclusters (GNCs) were anchored on the surface of rGO. These as-prepared composites kept their size, morphology, and fluorescent properties of original nanoparticles for FL imaging (40). 
The above-mentioned strategy is based on noncovalent binding between GO/rGO with inorganic nanomaterials. Likewise, a number of drugs (doxorubicin (DOX) (41), camptothecin (CPT) (42), etc.), photosensitizers (PSs) (chlorin e6 (Ce6) (17), sinoporphyrin sodium (DVDMS) (43), etc.) can be effectively loaded onto the surface of graphene, rGO or GO sheets via hydrophobic interactions and $\pi-\pi$ stacking. Besides the noncovalent binding, the covalent binding is also employed to modify the surface of graphene and its derivatives, which is usually achieved through the reaction with the $-\mathrm{COOH}$ and $-\mathrm{OH}$ groups (13). For instance, some dyes (Cy7 (44), Cy5 (45), IRDye800 (46), fluorescein (47), etc.), nanoparticles (UCNPs $\left.\left(\mathrm{Tm}^{3+} / \mathrm{Er}^{3+} / \mathrm{Yb}^{3+}\right)\right)(48)$, and biomolecules (folic acid (17), RGD peptide (49), Herceptin (50), transferrin (51), hyaluronic acid (52), $\beta$-cyclodextrin (53), TRC105 (54), vascular endothelial growth factor (VEGF) (55), etc.) were covalently linked to GO/rGO for FL imaging and active targeting, respectively. Meanwhile, this approach was also employed for radionuclide labeling, such as ${ }^{125} \mathrm{I}(44),{ }^{64} \mathrm{Cu}(54),{ }^{66} \mathrm{Ga}(56),{ }^{198,199} \mathrm{Au}(57),{ }^{111} \mathrm{In}(58)$, and so on. The radionuclidelabeled GOs can be used for PET/SPECT imaging.

There are also challenges facing the binding method: i) how to obtain GOs with uniform size and narrow distribution? ii) how to control the binding efficiency, density, and location of compounds/nanomaterials on $\mathrm{GO} / \mathrm{rGO}$ ? iii) how to improve the stability of graphene-based nanomaterials, especially for radionuclide labeling in PET/SPECT imaging? iv) how to control 
the distance between fluorescent components and $\mathrm{GO} / \mathrm{rGO}$, especially for fluorescent labeling in

FL imaging? It is crucial to avoid FL quenching of fluorophores by GO and keep the original fluorescent property of fluorophores for better performance in FL imaging.

\subsection{Surface functionalization}

Water dispersibility of graphene and reduced GO is rather poor due to the absence of oxygen-containing hydrophilic groups (59). Additionally, due to the charge screening effect, water-soluble GO tend to form aggregates in physiological buffers (13). Moreover, a number of studies have indicated that graphene and GO could cause toxicity in biological systems, strongly depending on their surface chemistry (60). Therefore, the surface functionalization of graphene and its derivatives is a crucial step for further biomedical applications. So far, two main strategies including covalent and non-covalent approaches, using various biocompatible coatings and targeting ligands, have been employed to engineer functionalized graphene-based materials with improved aqueous solubility, biocompatibility, and selectivity (49). For example, a variety of biocompatible and biodegradable macromolecules, such as polyethylene glycol (PEG),

polyvinylpyrrolidone (PVP), chitosan, and so on, have been used to modify graphene and its derivatives (26). Furthermore, a variety of biomacromolecules, such as deoxyribonucleic acids 
(DNAs), enzymes and proteins, were also employed to biofunctionalize graphene and its derivatives (26).

\section{Graphene-based nanomaterials in bioimaging}

\subsection{Optical imaging}

Optical imaging, a non-invasive technique, uses visible light and the special properties of photons to obtain detailed images of organs and tissues as well as smaller structures including cells and even molecules (24). It has advantages over other imaging modalities including relatively low cost, high sensitivity $\left(\sim 10^{-9}-10^{-12} \mathrm{~mol} / \mathrm{L}\right)$, nonionizing radiation, real-time imaging; short acquisition time, and multiplexing capability (61). However, this modality suffers from poor tissue penetration $(0-2 \mathrm{~cm})$, strong tissue scattering of photons in the visible light region (395-600 nm) (62), and significant background because of tissue autofluorescence and light absorption by proteins $(257-280 \mathrm{~nm})$, heme groups (absorbance maximum at $560 \mathrm{~nm}$ ), and even water (above $900 \mathrm{~nm}$ ) (63). To address these issues, near-infrared window (NIR, 650-900 nm)

(64) and second NIR window (NIR-II, 1000-1700 nm) $(65,66)$ imaging modalities have been explored with the advantages of reduced autofluorescence, reduced tissue scattering, and greater depth of penetration for in vivo imaging. 
Graphene-based nanomaterials were actively explored for optical imaging, mainly including FL imaging, two-photon FL imaging (TPFI), Raman imaging, and so on. The dyes, PSs, QDs, GNCs, or UCNPs functionalized GO/rGO have been widely investigated for FL imaging (12). GQDs, due to their intrinsic photoluminescence, can be used as photoluminescence probes for FL imaging. Nitrogen-doped GQDs (N-GQDs) exhibit a strong two-photon absorption cross section as high as 48000 Göppert-Mayer units and are demonstrated as an efficient two-photon fluorescent probe for TPFI (67). More interestingly, graphene and its derivatives also display great potential as efficient quenchers in graphene-based nanosensors for many fluorescent moieties, including small molecule dyes, QDs and conjugated polymers via FL resonance energy transfer or charge transfer (68). Additionally, the intrinsic Raman signals of GOs, $\mathrm{G}$ line (the $\mathrm{E}_{2 \mathrm{~g}}$ mode of $\mathrm{sp}^{2}$ carbon atoms) around $1600 \mathrm{~cm}^{-1}$ and the $\mathrm{D}$ line (the symmetry $\mathrm{A}_{1 \mathrm{~g}}$ mode) around $1350 \mathrm{~cm}^{-1}$, can be further enhanced by metal nanoparticles (Au or $\mathrm{Ag}$ ) for surface-enhanced Raman scattering (SERS) application in Raman imaging (32).

\subsubsection{Fluorescence imaging}

Fluorescence imaging is a non-invasive technique based on photons emitted from fluorescent probes $(24,69,70)$. Based on the intrinsic photoluminescence of nano-graphene 
oxide (nGO), the Dai group for the first time reported B-cell specific antibody Rituxan (antiCD20) conjugated and polyethylene glycol (PEG) modified GO (nGO-PEG-Rituxan) for Raji Bcells targeted FL imaging using an InGaAs detector under the $658 \mathrm{~nm}$ laser excitation (71). However, the quantum yield of nGO-PEG FL is rather low, which limits its further in vivo FL imaging of animals. Therefore, a number of groups utilized organic fluorescent dyes to functionalize $\mathrm{GO} / \mathrm{rGO}$ for in vitro and in vivo FL imaging. For instance, Liu group developed a NIR dye, Cy7, conjugated nGO-PEG (nGO-PEG-Cy7) for in vivo FL imaging of tumor xenografted mice, showing high tumor accumulation of nGO-PEG-Cy7 based on the enhanced permeability and retention (EPR) effect of cancerous tumors (44). Chen group developed a multifunctional VEGF-loaded IRDye800-conjugated graphene oxide (GO-IRDye800-VEGF) for FL imaging of ischemic muscle tissues in the murine hindlimb ischemia model (46). The fluorescence intensity of ischemic limbs is stronger than that of non-ischemic limbs at all tested time points after intravenous administration, suggesting that GO-IRDye800-VEGF could actively target ischemic muscle, likely owing to the increased permeability of blood vessels in hypoxic tissues. For dye labeling of GO/rGO, the "bridge" linker between fluorescent dyes and $\mathrm{GO} / \mathrm{rGO}$, such as PEG, is crucial to prevent FL quenching of fluorophores with graphene sheets (13). 
Besides conjugation with organic fluorescent dyes, the direct loading of dyes or PSs on GO/rGO was also investigated for FL imaging. Unfortunately, the FL of Ce6 (17) or 2-(1hexyloxyethyl)-2-devinyl pyropheophorbide-alpha (HPPH or Photochlor $\left.{ }^{\circledR}\right)$ (72), was drastically quenched by folic acid-conjugated GO and GO-PEG, respectively. Because both Ce6 and HPPH with single porphyrin ring allow them to be in good contact with the plane of GO via strong $\pi-\pi$ stacking and hydrophobic interactions, and form tight complexes with GO, leading to effective FL quenching via an energy/charge transfer process. The FL quenching limits these systems for photodiagnostics especially in FL imaging. To solve this problem, a photo-theranostic agent based on sinoporphyrin sodium (DVDMS) loaded PEGylated GO (GO-PEG-DVDMS) with improved FL property for enhanced optical imaging guided photodynamic therapy (PDT) was developed (Fig. 2A) (43). The FL of loaded DVDMS is drastically enhanced via intramolecular charge transfer. The emission peaks of DVDMS in GO-PEG-DVDMS are shifted to 644 and 670 nm (Fig. 2B). The FL intensities of GO-PEG-DVDMS are about 3-8 times higher than that of DVDMS at different weight ratios of GO-PEG: DVDMS (0.1:1-2:1) (Fig. 2C). As shown in Fig. 2D, fluorescence signal was mostly observed in the tumor area of mice intravenously (i.v.) injected with GO-PEG-DVDMS. As a comparison, free DVDMS group showed high signal throughout the body, especially in the skin. The GO-PEG-DVDMS appears to have potential for 
real-time FL visualization of in vivo DVDMS delivery and distribution, and imaging-guided PDT.

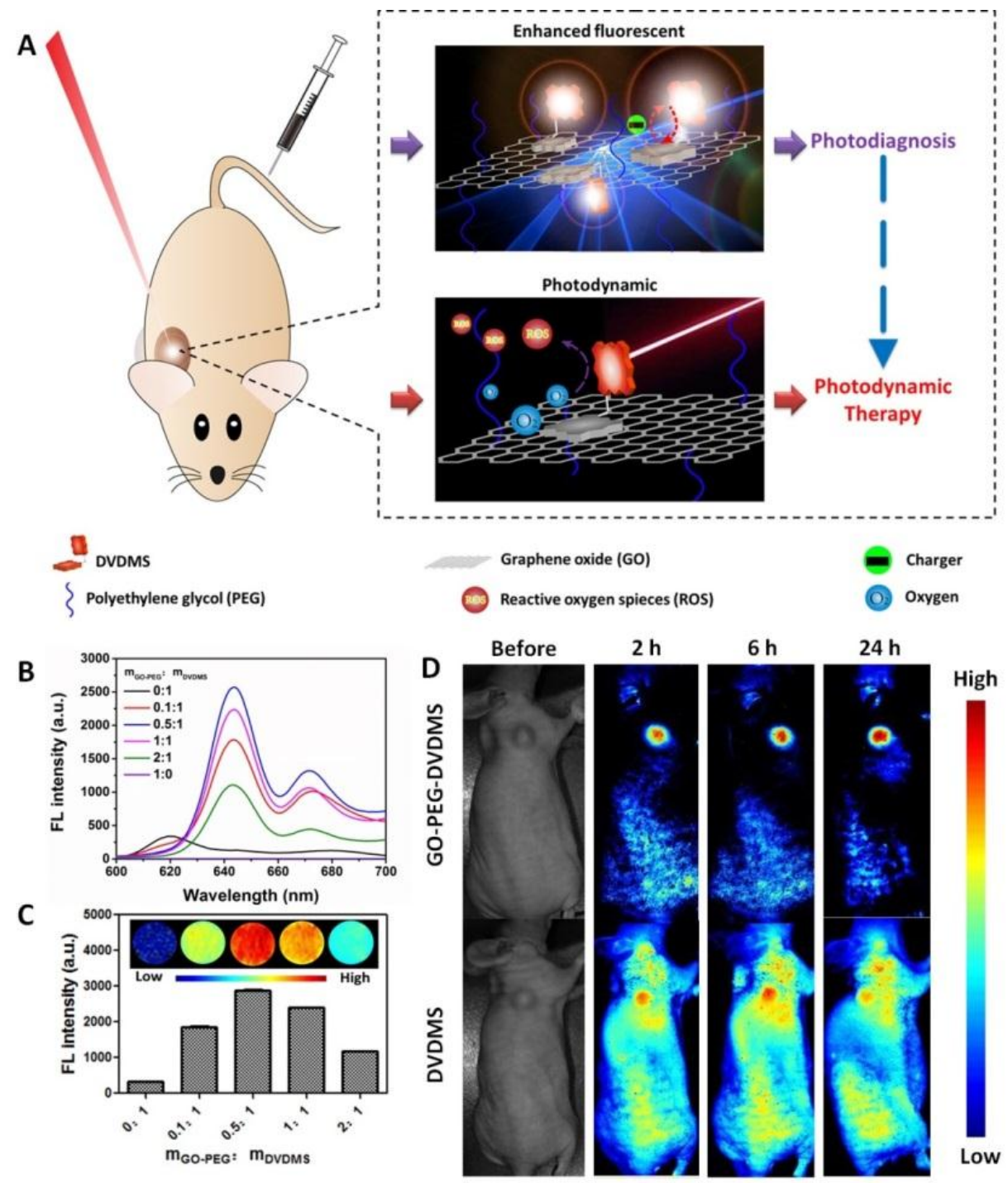

Fig. 2. (a) Sinoporphyrin sodium (DVDMS) loaded GO for enhanced FL imaging guided photodynamic therapy. (b) FL spectra of GO-PEG-DVDMS at different weight ratios of GO-PEG: DVDMS. DVDMS at $100 \mathrm{mg} / \mathrm{mL}$, GO-PEG at 10, 50, 100 and $200 \mathrm{mg} / \mathrm{mL}$. (c) FL intensity of DVDMS at $620 \mathrm{~nm}$ and different mix ratios by GO-PEG-DVDMS at $640 \mathrm{~nm}$. (d) In vivo FL imaging of mice bearing U87MG tumors 
intravenously (i.v.) injected with GO-PEG-DVDMS or DVDMS (DVDMS $2 \mathrm{mg} / \mathrm{kg}$ ) at different time points. Adapted and reproduced with permission from (43), Copyright 2015, Elsevier.

Due to the potential photobleaching of fluorescent dyes or PSs, inorganic nanomaterials like QDs, GNCs, or UCNPs have been used to integrate with GO/rGO for FL imaging. Chen et al. developed a multifunctional, low-toxicity QD-tagged rGO (QD-rGO) based on electrostatic interactions of positively charged poly(L-lysine)-rGO and negatively charged MUA capped$\mathrm{CdSe} / \mathrm{ZnS}$ QDs (38). The QD-rGO can serve as an imaging agent in the visible region and as a photothermal agent in the NIR region. This design overcomes the following limitations: the QD toxicity is mitigated by a surfactant coating, and the FL quenching is greatly reduced by introducing MUA and poly(L-lysine), as spacers between QDs and rGO. Wang et al. successfully anchored GNCs on rGO (GNC-rGO) which retained the NIR fluorescence property of GNCs for cellular imaging (40). Zhang et al. developed a multifunctional nanoplatform by covalently grafting core-shell structured UCNPs with nGO via PEG, and then loading phthalocyanine (ZnPc) on the surface of nGO (48). The UCNP-nGO/ZnPc nanoplatform was used for diagnosis as an upconversion luminescence (UCL) imaging probe of cells and whole-body animals with high contrast. 
Photoluminescent GQDs, composed of graphene lattices inside the dots, with good photostability, non-toxicity and easy functionalization, have been widely explored for FL imaging through quantum confinement and edge effects (23). The FL mechanism of GQDs may be originated from quantum size effect, electron hole recombination, zigzag sites and defect effect (energy traps). Nahain et al. developed GQD-hyaluronic acid (GQD-HA) with an average size of $20 \mathrm{~nm}$ for efficient CD44 targeted delivery to tumor-bearing balb/c female mice, demonstrating bright FL from the tumor tissue (73). The chemotherapy was achieved by releasing doxorubicin under mildly acidic conditions, which was loaded onto the basal plane of GQDs. In 2014, Ge et al. successfully synthesized a few GQDs with a broad absorption spanning the UV region and the entire visible region and strong deep-red emission peak at $680 \mathrm{~nm}(74)$. Both in vitro and in vivo studies clearly demonstrated that GQDs can act as PDT agents with a superior singlet oxygen quantum yield ( 1.3), photo- and pH-stability, as well as FL contrast agents for in vivo FL imaging. GQDs are good FL contrast candidates for FL imaging. For further bioimaging applications of GQDs, the quantum yield of GQDs still needs to be improved. Meanwhile, further surface modification is also necessary to enhance the optical property of GQDs, and improve the tumor accumulation rate and reduce reticuloendothelial system (RES) retention. 


\subsubsection{Two-photon fluorescence imaging (TPFI)}

Two-photon FL imaging (TPFI) has attracted much attention for its promising applications in both basic research and biomedical diagnostics, owing to the minor autofluorescence background, larger imaging depth (because of low Rayleigh scattering and low tissue absorption of NIR light), reduced photobleaching and phototoxicity than single-photon fluorescence imaging (12). It can provide more detailed analysis of various cellular/subcellular activities in the deep location of biological samples. Compared to one-photon excitation using simple continuous-wave lasers, two-photon nonlinear excitation requires a high reflux of excitation photons, usually by a femtosecond laser. The nonlinear excitation mode generates relatively high level of spatial resolution and decreases photobleaching. Furthermore, the twophoton excitation wavelength is known to be in the range of 700-1350 nm, it's quite suitable for imaging deep-sited organs and tissues.

Recently, carbon-based nanomaterials, including carbon dots (C-Dots), GO, and GQDs are attracting considerable interest in the field of TPFI. Wang and Gu et al. first reported transferrin functionalized GO-PEG with strong two-photon luminescence (TPL) as a nonbleaching optical probe for three-dimensional TPFI and laser-based cancer microsurgery, using an ultrafast pulsed laser as the excitation source (51). After that, Gong group developed N-GQDs as efficient two-photon fluorescent probes for cellular and deep-tissue imaging (Fig. 3) (67). The 
N-GQDs with an average size of $\sim 3 \mathrm{~nm}$ were prepared by a facile solvothermal method using dimethylformamide as both solvent and nitrogen source. Fig. 3A shows chemical structure of NGQDs with dimethylamine binding to GO that enables the extraction of smaller $s p^{2}$ domains from the large GO sheet. As shown in Fig. 3B, upon $800 \mathrm{~nm}$ femtosecond pulse laser excitation, the maximum two-photon FL emission wavelength of N-GQD is indistinguishable with that of the one-photon FL spectrum of N-GQD, but the bandwidth is much narrower. The inset of Fig. 3B is the two-photon FL image of the dried N-GQD samples. Fig. 3C displays the setup used for TPFI of N-GQDs in tissue phantom with different thicknesses. As shown in Fig. 3D, even at the depth of $1800 \mu \mathrm{m}, \mathrm{N}$-GQDs in the tissue phantom can still be identified with appreciable TPL signal, although the FL intensity is dramatically decreased. However, the OPFI (left panel of Fig. 3D) shows the maximum penetration depth of only $400 \mu \mathrm{m}$. Obviously, the TPFI using N-GQDs as fluorescent probe is particularly suitable for in vivo investigation of biostructures in the 800$1500 \mu \mathrm{m}$ region. 


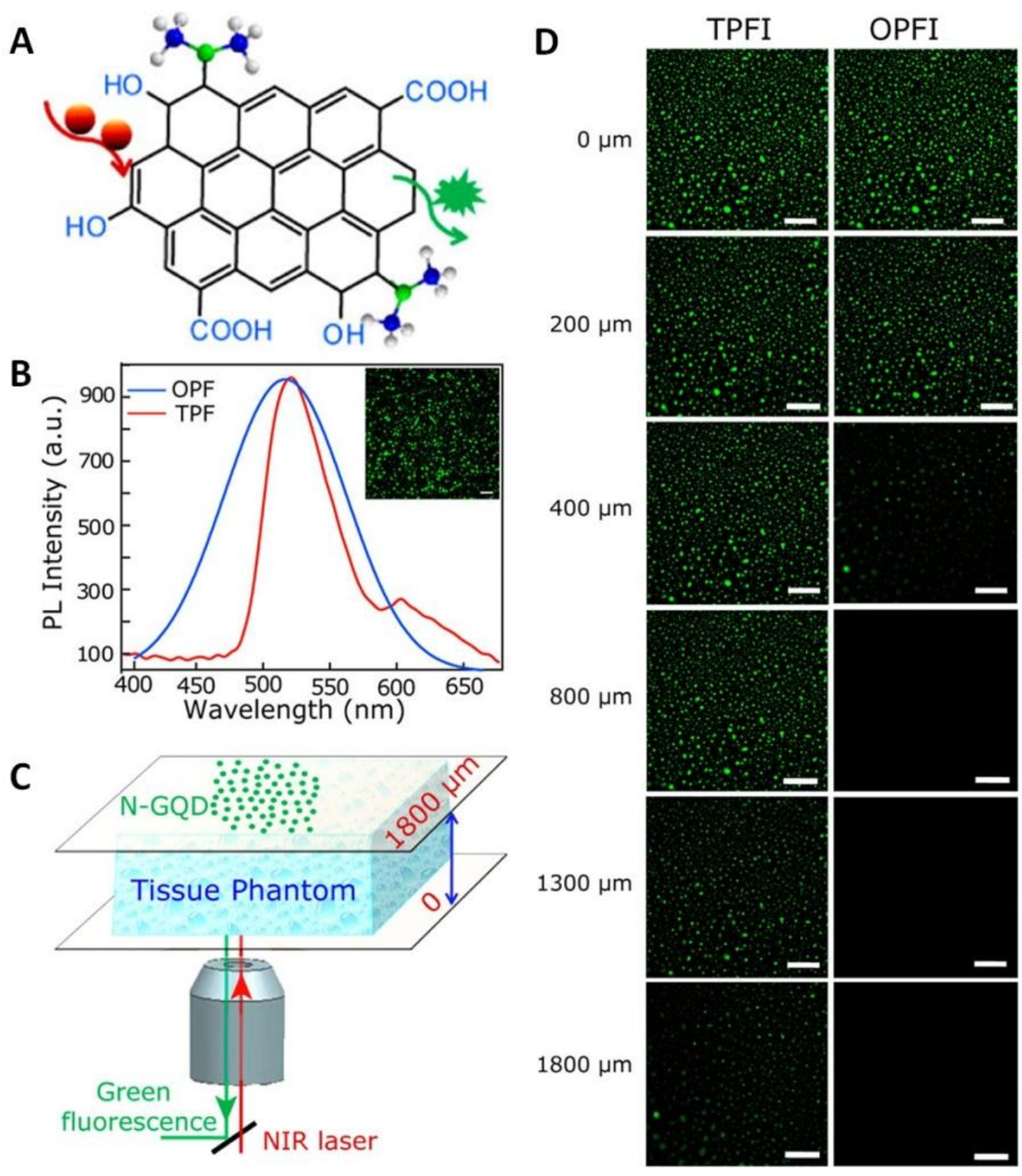

Fig. 3. Nitrogen-doped graphene quantum dots (N-GQDs) as efficient two-photon fluorescent probes for cellular and deep-tissue imaging. (A) Schematic illustration of N-GQDs chemical structure. (B) Twophoton-induced FL spectrum of N-GQD solution under $800 \mathrm{~nm}$ femtosecond laser excitation. Inset: Twophoton FL image of solid N-GQD (scale bar: $10 \mu \mathrm{m}$ ). (C) Schematic of the setup used for two-photon FL imaging (TPFI) of N-GQDs in tissue phantom with different thickness. (D) Penetration depth of N-GQDs 
for TPFI (right panel) and OPFI (left panel) in tissue phantom (all scale bar: $100 \mu \mathrm{m}$ ). Adapted and reproduced with permission from (67), Copyright 2013, American Chemical Society.

\subsubsection{Raman imaging}

Raman spectroscopy exploits the inelastic scattering of phonons derived from molecular vibrational excitation modes, which is different from the phonon absorption and emission in FL (12). Raman imaging offers a powerful analytical tool that extends the possibilities of vibrational spectroscopy with extremely high signal-to-noise ratio, negligible photo-bleaching, and multiplexing capabilities to solve chemical and biochemical problems in a non-destructive and non-perturbing manner. GOs as Raman tags exhibit intrinsically strong D and G peaks, which can be further enhanced by integrating metal nanoparticles (75-77). By in situ reduction of $\mathrm{Ag}^{+}$ on $\mathrm{GO}$, the $\mathrm{Ag} / \mathrm{GO}$ hybrids displays a singular remarkable surface enhanced Raman scattering effect (32). Folic acid conjugated Ag/GO hybrids were also developed for targeted SERS imaging on folate receptor (FR) positive cancer cells (75). By in situ reduction of $\mathrm{AuCl}_{4}^{-}$on $\mathrm{GO}$, Au/GO hybrids incubated Hela 229 cells exhibit much stronger Raman signals and more distinguishable Raman images than cells incubated with the pristine GO. Ma et al. reported gold nanoparticles compactly wrapped within nanosized GOs (NGO) as Raman imaging probe and a drug delivery system (78). The D and G peaks of Au@NGOs were about one order of magnitude 
stronger than those of the NGO, revealing sensitive imaging of internalized Au@NGOs in Hela cells. Liz-Marzán group successfully developed a method of seed-mediated growth of reduced graphene oxide-gold nanostar (rGO-NS) nanocomposites and employed them as active SERS materials for anticancer drug doxorubicin (DOX) loading and release (79). The rGO-NS nanohybrid shows tunable optical properties by simply changing the condition of growth reaction, improved stability as compared to bare Au nanostars, and sensitive SERS response toward aromatic organic molecules. Furthermore, SERS applications of rGO-NS to probe DOX loading and $\mathrm{pH}$-dependent release are successfully demonstrated.

Besides in situ synthesis of Au/GO hybrids, Zhang group developed Au/GO and Au/rGO composites by noncovalent attachment of $\mathrm{Au}$ nanoparticles pre-modified with 2mercaptopyridine to GO and rGO sheets, respectively, via $\pi-\pi$ stacking and other molecular interactions $(36,80)$. They demonstrated that the Au/GO hybrids are better SERS substrates than the Au NPs. Afterwards, the same group also loaded $20 \mathrm{~nm}$ of Au nanoparticles on the surface of sub-200 nm of GOs via amide coupling between PEG and DMSA ligands. The prepared Au/GO hybrids were served as Raman imaging probes for studying cellular uptake mechanisms in $\mathrm{Ca}$ Ski cell line.

Tan group reported the facile synthesis of alkyne-PEG-functionalized $\mathrm{AgCu} @$ graphene (ACG) nanoparticles (NPs) by using chemical vapor deposition (CVD) method to grow a few 
layers of graphene on the surface of AgCu NPs (81). The ACG NPs have been utilized to enhance the unique Raman signals from the graphitic shell, allowing ACG for cell labeling, Raman imaging, and SERS detection. As shown in Fig. 4A and B, the alkyne molecule diphenylacetylene, with a high Raman scattering cross section was efficiently conjugated on the PEG chain. Alkyne-PEG was functionalized on the surface of ACGs through strong $\pi-\pi$ stacking of diphenylacetylene tail and graphene. Compared with the Raman spectrum of alkyne-PEG, the results clearly demonstrate the successful functionalization of alkyne-PEG on the surface of ACGs (Fig. 4C). High-resolution Raman images of MCF-7 cells treated with functionalized ACGs are shown in Fig. 4D-H. All three modes, i.e., D, G, and alkyne, were utilized for the colocalization of ACGs, demonstrating good intracellular localization capability. All signals spread throughout the cell cytoplasm and accurately pinpoint the location of ACGs. 

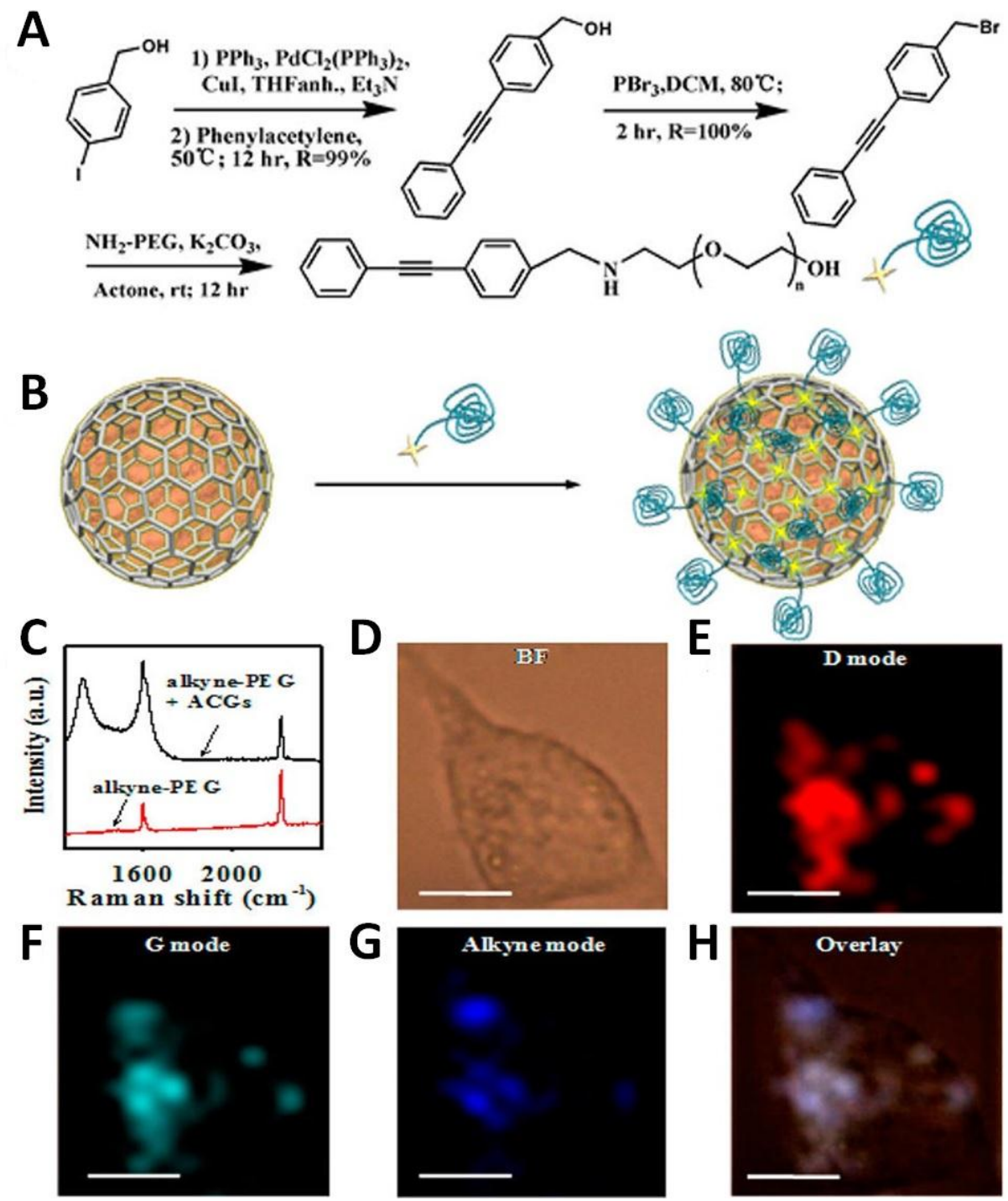

Fig. 4. (A) Synthesis procedure of alkyne-PEG. (B) Schematic illustration of the alkyne-PEG functionalization of ACGs. (C) Raman spectra of alkyne-PEG with (black) and without (red) ACGs. (D-H) Raman image of MCF-7 cells treated with alkyne-PEG-modified ACGs using $1 \mathrm{~s}$ integration/pixel. BF, bright field; scale bar, $10 \mu \mathrm{m}$. Adapted and reproduced with permission from (81), Copyright 2014, American Chemical Society. 


\subsection{Radionuclide-based imaging}

FL imaging cannot provide quantitative results and sometimes may be interfered by FL quenching or photo-bleaching of fluorescent dyes, light absorption and scattering of tissues, and autofluorescence background. Radiolabeling method would be able to accurately track the labeled substances in vivo in a quantitative manner with excellent sensitivity $\left(\sim 10^{-11}-10^{-12} \mathrm{~mol} / \mathrm{L}\right)$ and limitless penetration depth (24). The radionuclide-based imaging mainly contains PET and SPECT. PET and SPECT images are acquired over a nominally low background signal and require little signal amplification (63). Graphene-based nanomaterials as promising nanoplatforms are playing an important role in PET/SPECT imaging.

In 2011, Liu group developed a method to label nGO-PEG with ${ }^{125}$ I by anchoring iodine atoms on the defects and edges of GO (44). Based on this method, a number of studies have been explored. Cai and co-workers demonstrated in vivo active tumor targeting using ${ }^{64} \mathrm{Cu}$ labeled nGO-PEG (54). The in vivo PET imaging results evidenced the active tumor targeting by conjugating nGO-PEG with an antibody, TRC105, to target vasculatures in 4T1 murine breast tumors. As shown in Fig. 5A, the GO conjugates was labeled with ${ }^{64} \mathrm{Cu}$ through $1,4,7-$ triazacyclononane-1,4,7-triacetic acid (NOTA). The different time points of postinjection (p.i.) were chosen for serial PET scans. The PET imaging results demonstrated CD105 specific 
targeting of TRC105 conjugated GO (Fig. 5B). A parallel study used ${ }^{66} \mathrm{Ga}$ labeled nGO-PEG for tumor-targeted PET imaging (56).

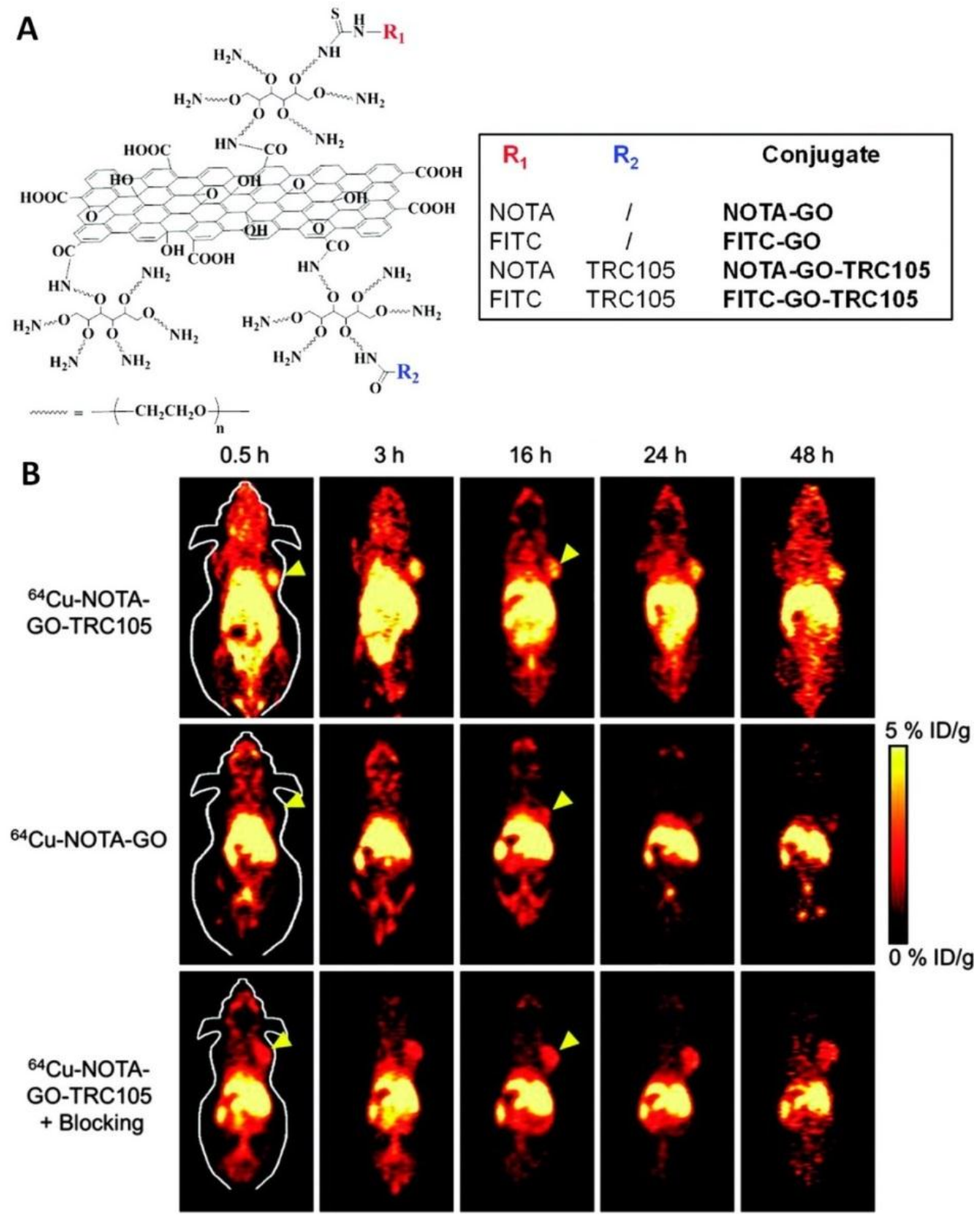

Fig. 5. In vivo PET imaging of radiolabeled nGO-PEG. (A) A scheme of nGO-PEG conjugated with anti-CD105 antibody (TRC105) and 1,4,7-triazacyclononane-1,4,7-triacetic acid (NOTA). The latter was 
used for ${ }^{64} \mathrm{Cu}$ labeling. (B) Time-dependent in vivo PET imaging of mice bearing 4T1 tumors i.v. injected with ${ }^{64} \mathrm{Cu}$-NOTA-nGO-TRC105, ${ }^{64} \mathrm{Cu}-\mathrm{NOTA}-\mathrm{nGO}$, and ${ }^{64} \mathrm{Cu}-\mathrm{NOTA}-\mathrm{nGO}-\mathrm{TRC} 105$ after a preinjected blocking dose of TRC105. Tumors are indicated by arrowheads. Adapted and reproduced with permission from (54), Copyright 2012, American Chemical Society.

In comparison to PET, SPECT is approximately ten times less sensitive $\left(\sim 10^{-10}-10^{-11}\right.$ mol/L); however, SPECT enables concurrent imaging of multiple radionuclides of different energies (61). Additionally, SPECT, without the need of cyclotron, is more widely available than PET in the clinic, and SPECT radionuclides are simple to prepare and usually have a longer halflife than PET radionuclides. Cornelissen et al explored the use of anti-HER2 antibody (trastuzumab)-conjugated $\mathrm{nGO}$, radiolabeled with ${ }^{111}$ In-benzyl-diethylenetriaminepentaacetic acid (BnDTPA) via $\pi-\pi$ stacking, for in vivo targeting and SPECT imaging (58). Radiolabeled nGO-trastuzumab conjugates demonstrated better pharmacokinetics compared to radiolabeled trastuzumab without NGO, with more rapid clearance from the circulation. Fazaeli et al. investigated the ${ }^{198,199} \mathrm{Au} @ \mathrm{AF}-\mathrm{GO}$ nanostructure for in vivo targeting and SPECT imaging of tumors (57). The ${ }^{198,199} \mathrm{Au} @ \mathrm{AF}-\mathrm{GO}$ had excellent tumor targeting/imaging and fast washing out from the body. 


\subsection{Magnetic resonance imaging (MRI)}

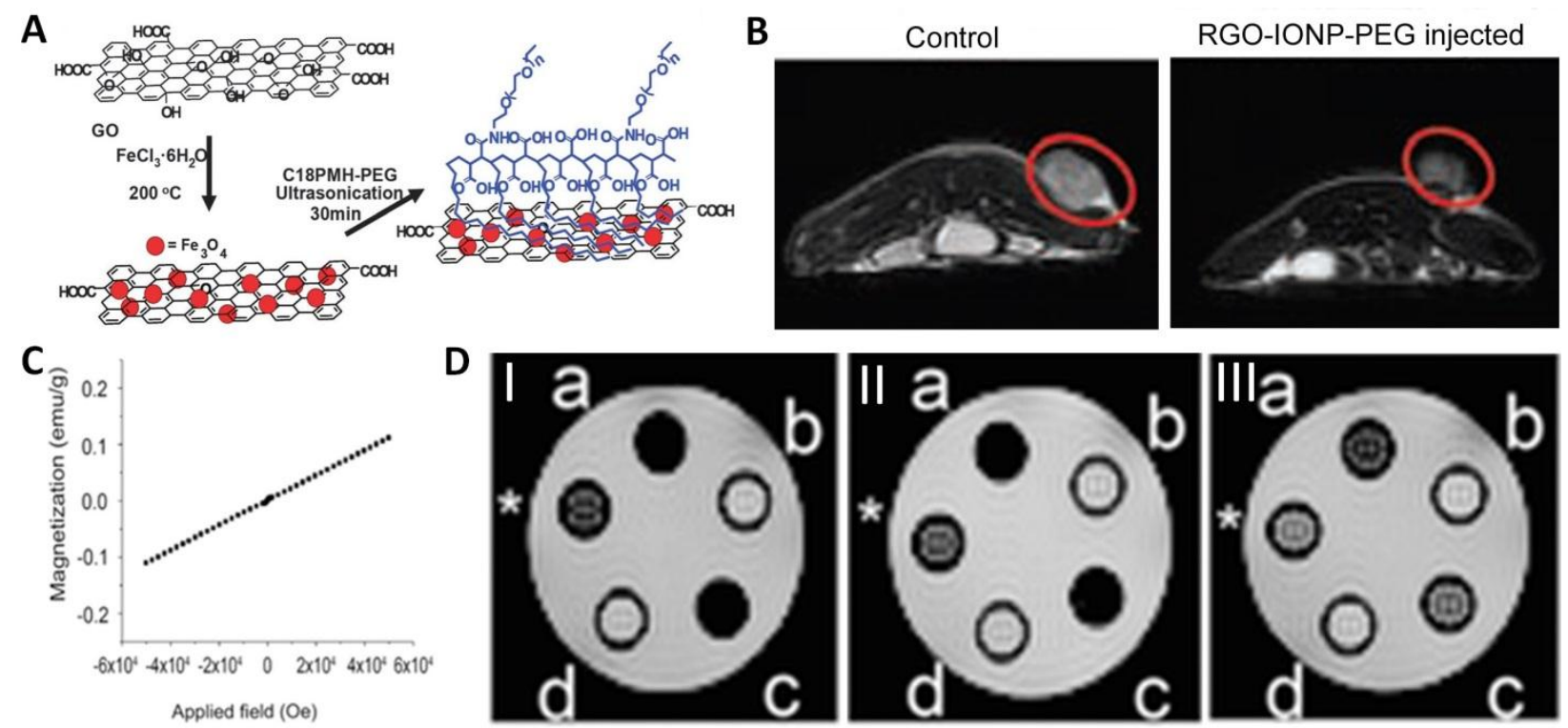

Fig. 6. (A) A scheme showing preparation and functionalization of PEGylated RGO-IONP nanocomposites (RGO-IONP-PEG). (B) In vivo MRI of 4T1-tumor bearing mice using RGOIONP-PEG as the contrast agent. (C) SQUID magnetization curve of fluorinated graphene oxide (FGO). (D) Spin-spin ( $\left.T_{2}\right)$ relaxation measurements obtained on a $4.7 T$ MRI for FGO (I: a = 625, $\mathrm{c}=500$; II: $\mathrm{a}=313, \mathrm{c}=250$; III: $\left.\mathrm{a}=156, \mathrm{c}=125 \mu \mathrm{g} \mathrm{mL} \mathrm{mL}^{-1}\right)$ and GO (I: $\mathrm{b}=625, \mathrm{~d}=500$; II: $\mathrm{b}=313, \mathrm{~d}=250 ; \mathrm{III}: \mathrm{b}=156, \mathrm{~d}=125 \mu \mathrm{g} \mathrm{mL} \mathrm{L}^{-1}$ ) with the positive control $(*)$ consisting of diluted magnevist $\left(0.5 \mathrm{mg} \mathrm{mL}^{-1}\right)$. Adapted and reproduced with permission from (35), Copyright 2012, Wiley-VCH (a and b). Adapted and reproduced with permission from (82), Copyright 2013, Wiley-VCH (c and d). 
Compared to optical and radionuclide imaging, MRI, a noninvasive technique without ionizing radiation, has been widely used to image the anatomy as well as function of tissues in a quantitative manner with excellent spatial resolution. However, MRI suffers from its low sensitivity $\left(\sim 10^{-3}-10^{-5} \mathrm{~mol} / \mathrm{L}\right)$ and long signal acquisition time (24). Because the lattice-spin (longitudinal) relaxation time $T_{1}$ and spin-spin (transverse) relaxation time $T_{2}$ of water proton's magnetic moment are environment-dependent, MRI contrast agents have been actively explored to enhance the relaxivity for improving the $T_{1}$ or $T_{2}$ contrast in the observable water pool (83). $T_{1}$ agents are mainly $\mathrm{Gd}^{3+}$-based and provide brighter images, whereas $T_{2}$ agents produce darker images and are usually iron oxide nanoparticle (IONP).

Ions of paramagnetic metals such as gadolinium $(\mathrm{Gd})$ and manganese $(\mathrm{Mn})$ are generally toxic owing to the non-selective coordination with biomolecules (84). GOs with many oxygenated functional groups and cavities, can be readily coordinated with these ions by chelation or sequestering the ions between graphene layers, which mitigates the toxicity of these ions (12). For example, Gizzatov et al. chelated $\mathrm{Gd}^{3+}$ ions with carboxyphenylated graphene nanoribbons (GNRs) for enhanced MRI relaxivity $\left(r_{1}=70 \pm 6 \mathrm{mM}^{-1} \mathrm{~s}^{-1}\right.$ and $r_{2}=108 \pm 9 \mathrm{mM}^{-1} \mathrm{~s}^{-}$ ${ }^{1}$ ), which are 16 and 21 times greater than the current clinically available $\mathrm{Gd}^{3+}$-based $T_{1}$ agents

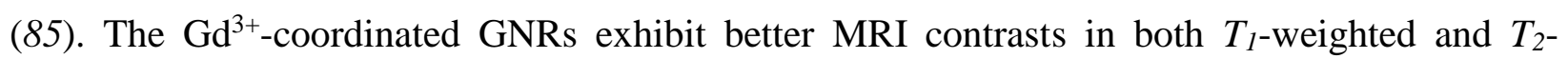
weighted images. Wei and Ma et al. developed poly(amidoamine) dendrimer-grafted 
gadolinium-functionalized nanographene oxide (Gd-NGO) nanoparticles as effective carriers to deliver both chemotherapeutic drugs and highly specific gene-targeting agents such as microRNAs (miRNAs) (86). Gd-NGO could be detected by MRI to identify the tumor area and quantify the concentration of therapeutics within the tumor.

Because IONP can be directly grown on graphene or its capped ligands can be linked with graphene, the superparamagnetic GOs/IONP hybrids were widely explored as $T_{2} \mathrm{MR}$ contrast agents (13). For instance, Zhang group developed a GO-based platform for the formation of aggregates of dextran-coated $\mathrm{Fe}_{3} \mathrm{O}_{4}$ NPs to induce efficient $T_{2}$ shortening (87). The dextran-coated $\mathrm{Fe}_{3} \mathrm{O}_{4}$ NPs can anchor onto graphene sheet to form clusters or aggregates, affording enhanced MRI contrast compared to the individual $\mathrm{Fe}_{3} \mathrm{O}_{4}$ NPs. Liu group prepared rGO/IONP nanocomposite starting from GO and iron chloride hexahydrate via a hydrothermal reaction (Fig. 6A) (35). The rGO/IONP was further functionalized with the amphiphilic $\mathrm{C}^{18} \mathrm{PMH}-\mathrm{PEG}$ polymer via hydrophobic interactions for in vivo MRI guided photothermal therapy (PTT) (Fig. 6B). Real-time monitoring of photothermal therapeutic response of tumors by MRI was also demonstrated. Fu et al developed GO/IONP as a theranostic agent to diagnose and treat regional lymph nodes (RLN) metastasis of pancreatic cancer (88). The GO/IONP is able to visualize the regional lymphatic system by dual-modality mapping of MRI, which is helpful for surgeon to make the preoperative plan. The dual-modality mapping is able to 
intraoperatively distinguish RLN from surrounding tissue, and then the metastatic lymph nodes could be effectively ablated upon laser irradiation.

Besides paramagnetic metal complexes and IONP, fluorinated graphene oxide (FGO) was also explored as non-magnetic-nanoparticle carbon-based MRI contrast agent by creating dipolar $\mathrm{C}-\mathrm{F}$ bonds as the paramagnetic centers (82). The superconducting quantum interference device (SQUID) characterization of the synthesized FGO showed a linear dependency with the magnetic field, suggesting its paramagnetic behavior (Fig. 6C). As shown in Fig. 6D, FGO showed good $T_{2}$ contrast.

\subsection{Photoacoustic imaging (PAI)}

The low-energy electromagnetic waves can penetrate deeper than the short wavelengths, radio frequency waves exhibit much lower scattering in the biological samples, which should be suitable for deep tissue/organ imaging. Photoacoustic imaging (PAI) is a hybrid imaging modality based on the PA effect, in which the absorbed short-pulsed electromagnetic energy (nonionizing laser pulses) is converted into heat, resulting in acoustic emission due to a transient thermoelastic expansion (89). In principle, upon pulsed laser irradiation, tissues or contrast agents absorb light and generate a pressure rise by localized thermoelastic expansion, then emit broadband acoustic waves during contraction that can be collected by ultrasound transducers and 
processed with similar reconstruction algorithms (90). PAI offers optical absorption contrast with the resolution of ultrasound for deep tissue/organ imaging.

In the graphene family nanomaterials (GFNs), rGO plays an important role as PA contrast agents, because rGOs with larger $s p^{2}$ domains than GOs, can absorb NIR light more efficiently (91). However, the hydrophilicity of GOs is decreased after the reduction, leading to poor water solubility of rGOs. To address this issue, many methods have been explored. Patel et al. reported direct production of less oxygenated nanosize graphene sheets, which have small lateral size $(\sim 10 \mathrm{~nm})$ and can be easily dispersed in water with higher absorption in the NIR region for PA imaging (92). To further enhance the absorption cross-section of GO, indocyanine green (ICG), with strong absorbance in the NIR region, was loaded onto GO by $\pi-\pi$ stacking interactions (93). The ICG-GO complex has a high optical absorbance in the NIR region, and promises as an ultrahigh sensitive PA contrast agent.

Besides, GOs can be reduced and stabilized by BSA via one-step reduction method to afford the nanosized, reduced graphene oxide (nano-rGO) with high stability and low cytotoxicity as reported by Cai group (Fig. 7A) (94). The BSA functionalized nano-rGO has small lateral size $(\sim 70 \mathrm{~nm})$ and good monodispersity. Fig. $7 \mathrm{~B}$ shows the photograph of the mouse bearing MCF-7 tumor xenograft. The site and boundaries of tumor was confirmed by ultrasound imaging (Fig. 7C). Before i.v. administration of nano-rGO, the PA image showed 
observable but weak PA signals in the tumor region (Fig. 7E). At $2 \mathrm{~h}$ after administration, the PA signal in the tumor was significantly increased (Fig. 7E). The subtracted image showed prominent PA signal from the tumor area, suggesting the high tumor accumulation of nano-rGO. Region of interest analysis revealed that the tumor PA signal peaked within $0.5 \mathrm{~h}$ and then plateaued for at least $4 \mathrm{~h}$ (Fig. 7D), suggesting BSA-functionalized nano-rGO as a good PA contrast agent.
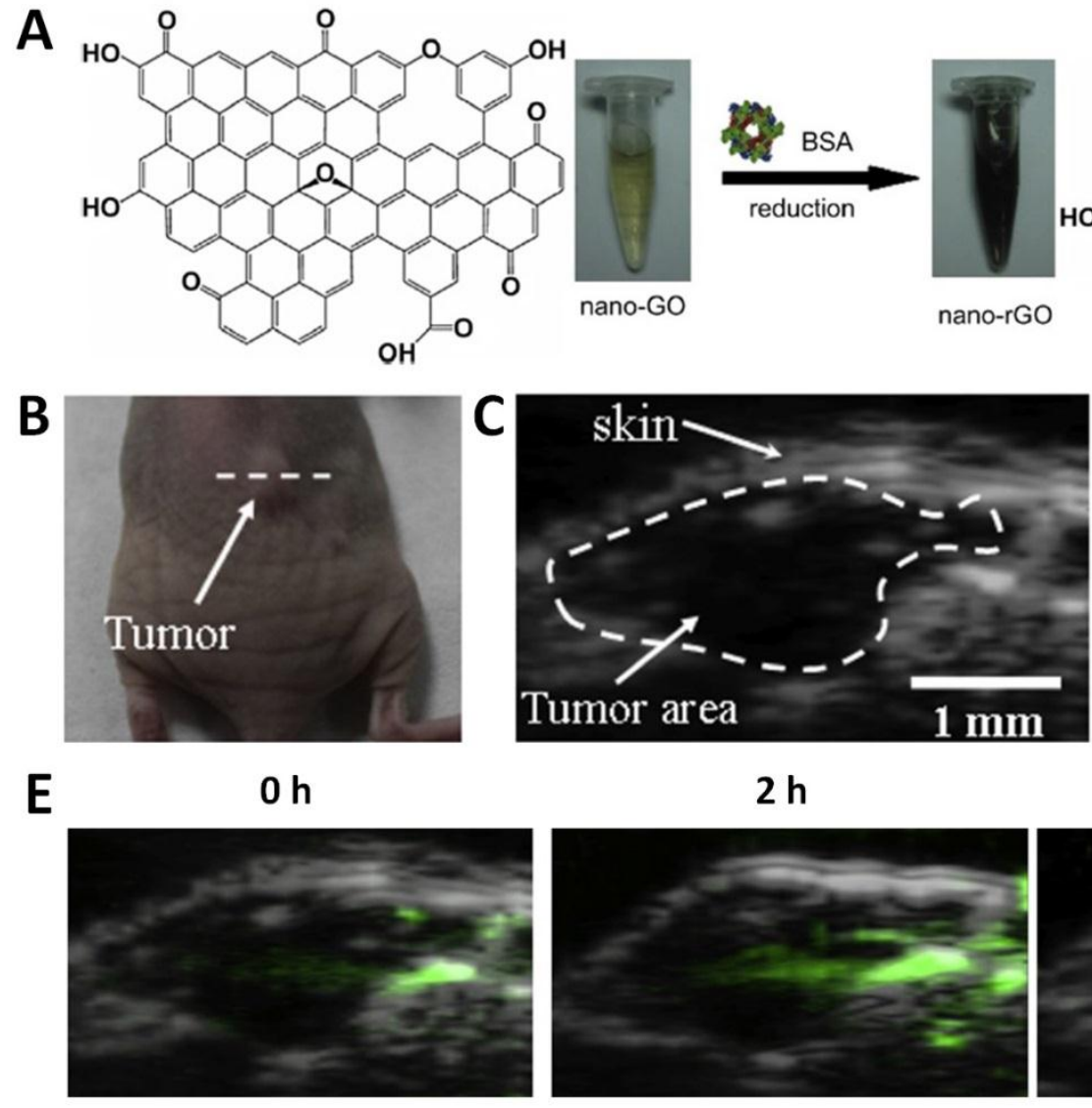

$2 \mathrm{~h}$

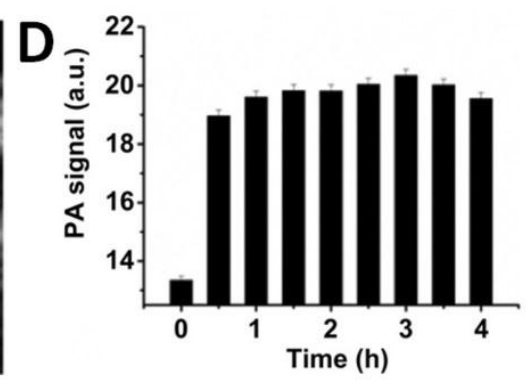

Subtraction imaging

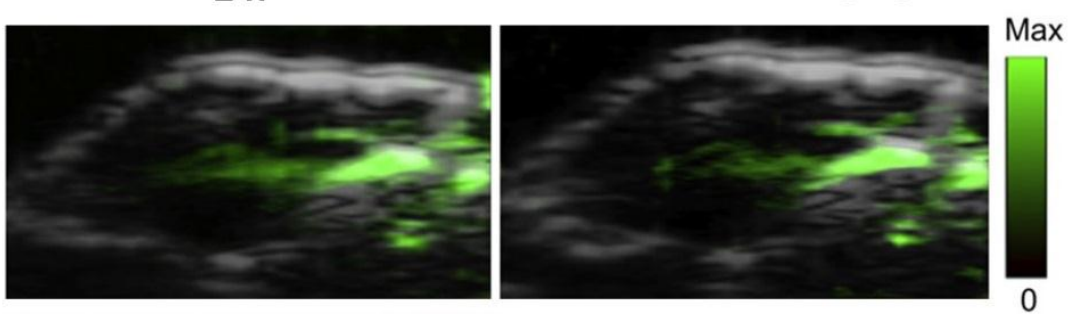

Fig. 7. (A) A scheme showing the preparation of nano-rGO from nano-GO using BSA as reductant and stabilizer. (B) Photograph of the mouse bearing MCF-7 tumor xenograft. (C) 
Ultrasound (gray) image of the tumor region. (D) PA signal in the tumor region as a function of the injection time. (E) Ultrasound and PA dual-modality images of the tumor region using nanorGO as contrast agent. Adapted and reproduced with permission from [84], Copyright 2013, Elsevier.

\subsection{Computed Tomography (CT)}

Computed tomography (CT) can provide complementary anatomical information (95).

CT measures the absorption of X-rays when they pass through targets. The ability of CT to distinguish tissues is based on the fact that different tissues provide distinct degrees of X-ray attenuation, where the attenuation coefficient depends on the atomic number and electron density of the tissues. Differences in absorption between bone, fat, air, and water produce high contrast images of anatomical structures.

Nanomaterials containing electron dense elements with high atomic number such as iodine, bismuth or gold have been proposed as CT contrast agents (96). CT contrast requires high concentrations of these elements. Zhang et al. used GO@ Ag nanocomposite as an X-ray contrast agent for X-ray imaging application (97). Wu and Yang et al. prepared $\mathrm{GO} / \mathrm{BaGdF}_{5} / \mathrm{PEG}$ nanocomposites using a solvothermal method for X-ray CT imaging of the tumor model in vivo, which shows better X-ray attenuation property than Iohexol (98). Liu (99) and Dai (100) groups used gold to endow GO the X-ray attenuation for X-ray and CT imaging, respectively. 


\subsection{Multimodal Imaging}

Recently, the idea of using multiple imaging modalities in conjunction has gained popularity (63). Multimodality imaging refers to integrating the merits of individual imaging modality, and collecting all information from different imaging modalities, which offers higher efficiency and accuracy of diagnosis $(101,102)$. Meanwhile, combination of multimodal detectability in a single agent would avoid the additional stress on the body's blood clearance that accompanies the administration of multiple doses of agents (103).

Graphene and its derivatives, due to their large surface area and versatile chemistry, have been utilized as building blocks for the construction of multimodal imaging contrast agents. Liu group developed a probe based on the intrinsic high NIR optical absorbance and strong magnetic property of RGO-IONP-PEG nanocomposite, as well as external labels for triple modal FL, PA, and MR imaging (35). In another case, they reported a dual-nanostructure modified graphenebased nanocomposite by growing a layer of gold on the synthesized GO-IONP, obtaining a GOIONP-Au nanocomposite, then functionalized with PEG for dual-modal MR and X-ray imaging (99). Recently, Wu and Yang et al. reported a structure with $\mathrm{BaGdF}_{5}$ NPs directly grown on the surface of GO nanosheets in the presence of PEG. The resulting GO-BaGdF5-PEG shows low cytotoxicity, positive MR contrast effect and better X-ray attenuation property than Iohexol, which enables effective dual-modality MR and X-ray CT imaging (98). 
Dai group developed poly(lacticacid)(PLA) microcapsules containing GO and AuNPs through a double-microemulsion water-in-oil-in-water (w/o/w) solvent evaporation technique combined with electrostatic layer-by-layer (LbL) technique for photothermal tumor destruction under the guidance of ultrasonic/CT bimodal imaging (100). The PLA microcapsule plays a dual role: it enhances ultrasound imaging and loads GO and Au NPs. Au NPs enhance CT contrast and GO serves as a strong NIR-light absorbing agent, which efficiently converts the absorbed light into heat.

We developed a nanoformula of GO-PEG loaded with photosensitizer HPPH via $\pi-\pi$ stacking for PDT of tumors (72). The FL property of HPPH was used for FL imaging. Moreover, we labeled HPPH with a positron emitting radioisotope, copper-64 $\left({ }^{64} \mathrm{Cu}, t_{1 / 2}=12.7 \mathrm{~h}\right)$, for PET imaging. The obtained GO-PEG-HPPH complex allows dual-modality FL and PET imaging. Recently, we used DVDMS-loaded GO-PEG (GO-PEG-DVDMS) for enhanced FL/PA dualmodal imaging and combined PDT and PTT (104). The GO-PEG carrier drastically improves the FL of loaded DVDMS via intramolecular charge transfer. Concurrently, DVDMS significantly enhances the NIR absorbance of GO for improved PAI and PTT. The as-prepared GO-PEGDVDMS is well suited for FL/PA dual-modal imaging and synergistic PDT/PTT.

\section{Prospects and challenges}


For bioimaging applications, the multiplex roles of graphene and its derivatives have been gradually uncovered: i) as imaging contrast agents due to their intrinsic FL emission, Raman scattering, and NIR absorbance (12); ii) as carriers because they have two accessible sides with large specific surface area (theoretical value of $2,630 \mathrm{~m}^{2} \mathrm{~g}^{-1}$ ) that offer high loading capacity of drugs, dyes, PSs and other inorganic nanomaterials by physical absorption, Van der Waals forces, electrostatic binding, or charge transfer interactions (105-108); iii) as fluorescence quenchers because their $s p^{2}$ carbon structure is able to quench small molecule dyes, QDs and conjugated polymers via FL resonance energy transfer or charge transfer (109-111); iv) as wrapping materials because their flexible and amphiphilic structures make them suitable for wrapping or encapsulating insoluble nanoparticles, thus improving their water solubility and stability, biocompatibility, and preventing aggregation, degradation or toxicity in biological systems (112-114); v) as building blocks. Their ultra-high surface area and versatile surface functionalization promise the synthesis of graphene-based composites, opening a new avenue for new materials construction (115-118).

The photoluminescence properties of graphene and its derivatives are different (12). For graphene, GO, and GQDs, their intrinsic photoluminescence is emitted with UV excitation, and tunable emission wavelength is located in the UV-Vis range (12). Their quantum yield (QY) is in the range of $0.02-70.3 \%$ (119-121). However, reduced GO shows a strong photoluminescence 
quenching effect with enhanced absorbance in the NIR region (12), unlike single-walled carbon nanotubes (SWNTs), which has unique photoluminescence in both NIR-I (700-900 nm) and NIR-II (1100-1400 nm) regions (122-124).

The intrinsic Raman characteristic vibrational modes of graphene and its derivatives are located in the range of 1000 3000 $\mathrm{cm}^{-1}$ : the D peak, the G peak, and the 2D peak $(125,126)$. The D peak (the symmetry $\mathrm{A}_{1 \mathrm{~g}}$ mode) at $1350 \mathrm{~cm}^{-1}$ belongs to the breathing mode. The $2 \mathrm{D}$ peak (also called as G0 peak) is located at about twice the frequency of the D peak. The G peak (the $E_{2 g}$ mode of $\mathrm{sp}^{2}$ carbon atoms) at $1600 \mathrm{~cm}^{-1}$ corresponds to the in-plane motion of the carbon atoms. For practical Raman imaging, the G peak is usually selected to determine the amount of graphene and its derivatives (12). Compared with SWNTs, it also has multiple Raman characteristic vibrational modes, including the radial breathing mode $\left(100-300 \mathrm{~cm}^{-1}\right)$ and the $\mathrm{G}$ band $\left(\sim 1580 \mathrm{~cm}^{-1}\right)$, which correspond to the vibration of carbon atoms in the radical direction and in the tangential direction, respectively (122). However, the resonance Raman scattering of SWNTs is determined by the density of states available for the optical transition (e.g. $E_{11}$ and $\mathrm{E}_{22}$ transition), which is dependent on its chirality and diameter (127). Unlike SWNTs, the Raman scattering of graphene and its derivatives is not chirality dependent, and the electronic structure of graphene with a small band gap allows a wide range of photons (visible to NIR) to be used for Raman imaging (12). 
The photoacoustic property of GOs and GQDs is usually suppressed due to their disconnected small $\mathrm{sp}^{2}$ domains with oxygenated functional groups (1). The transition energy from the highest occupied molecular orbital (HOMO) to the lowest unoccupied molecular orbital (LUMO) is higher than the larger $s p^{2}$ domains, resulting in low NIR absorbance. For reduced GO, their small fragments of $s p^{2}$ domains are reconnected with increased conductive and NIR absorbance (128). The extinction coefficient of rGO in the NIR region is comparable to that of SWCNTs, which promises efficient photothermal and photoacoustic properties for PTT and PAI.

To further improve the performance of graphene and its derivatives in bioimaging applications, it is essential to precisely control their sizes, components, and surface coatings so that batch to batch variation is minimized. For example, how to obtain GOs with uniform size and narrow distribution? Current synthesis methods are usually based on the top down strategy of stripping graphite. The bottom up strategy may have potential to make very uniform GOs from designed small molecules. Besides, the properties of contrast agents for different modalities are also very important. For example, how to control the distance between fluorescent components and $\mathrm{GO} / \mathrm{rGO}$ ? It is crucial to prevent FL quenching of fluorophores on graphene sheets. Additionally, how to improve the quantum yield of GOs and GQDs for FL imaging? For Raman imaging, how to enhance the Raman signals of GOs by metal nanoparticles? For PET/SPECT imaging, how to improve the radionuclide labeling efficiency and metabolic 
stability of the radiolabeled GOs? For MRI, how to improve the relaxivity of graphene-based MR contrast agents? For PAI, how to enhance the optical absorbance of graphene-based PA contrast agents in the NIR region? Overall, there is still a long way before the clinical translation of graphene-based contrast agents.

For biomedical applications of graphene-based nanomaterials, the concern of long-term toxicity is another major concern (59). Systematic assessment of their toxicity, biocompability, immunogenicity, pharmacokinetics, and biodistribution from small animal models (e.g. zebra fish, mice, etc.) to large animal models (e.g. pigs, dogs, monkeys, etc.) will be critical $(60,129)$. Furthermore, more knowledge of their biological behaviors is not only helpful to understand their toxicology, but also useful to improve their bioimaging performances. Optimization of graphene-based nanomaterials aims to improve the tumor accumulation rate with high bioavailability and reduce RES retention by using specific biomarkers (such as peptides, antibodies). Meanwhile, specific surface functionalization of graphene-based nanomaterials is highly desirable to across various biological barriers, such as blood brain barriers, extracellular matrix, mucus, and so on.

\section{Conclusions}


In this review, we summarized the recent progress of graphene-based nanomaterials as imaging contrast agents for different bioimaging applications. The dyes, PSs, QDs, GNCs, or UCNPs functionalized GO/rGO have been used for FL imaging. GQDs and their derivatives, due to their intrinsic photoluminescence, have also been employed for FL imaging and TPLI. GOs with intrinsically strong Raman signals of D and G peaks have been used for Raman imaging, which can be further enhanced by integrating metal nanoparticles ( $\mathrm{Au}$ or $\mathrm{Ag}$ ). Radionuclidelabeled GOs have been explored for PET/SPECT imaging. Ions of paramagnetic metals or IONP functionalized GO/rGO have been developed for MRI. The rGOs or dyes/PSs modified GOs with strong optical absorbance in the NIR region are suitable for PAI. Nanoparticles containing electron dense elements with high atomic number can be combined with GOs for X-ray or CT imaging. By integrating other materials with specific properties onto GOs, multimodal imaging can be achieved in one platform. It is anticipated that graphene-based nanomaterials will play significant roles in bioimaging and understand the fate of the related therapeutics.

Conflict of Interest: The authors declare no competing financial interest.

\section{Acknowledgements}


This work was supported by the National Science Foundation of China $(51573096,81401465)$, and the Intramural Research Program (IRP) of the NIBIB, NIH.

\section{References}

1. Loh KP, Bao Q, Eda G, Chhowalla M. Graphene oxide as a chemically tunable platform for optical applications. Nat Chem. 2010;2(12):1015-1024.

2. Geim AK, Novoselov KS. The rise of graphene. Nat Mater. Mar 2007;6(3):183-191.

3. Allen MJ, Tung VC, Kaner RB. Honeycomb carbon: a review of graphene. Chem Rev. Jan 2010;110(1):132-145.

4. Novoselov KS, Geim AK, Morozov SV, et al. Electric field effect in atomically thin carbon films. Science. Oct 22 2004;306(5696):666-669.

5. Dikin DA, Stankovich S, Zimney EJ, et al. Preparation and characterization of graphene oxide paper. Nature. Jul 26 2007;448(7152):457-460.

6. Dreyer DR, Park S, Bielawski CW, Ruoff RS. The chemistry of graphene oxide. Chem Soc Rev. Jan 2010;39(1):228-240.

7. Eda G, Fanchini G, Chhowalla M. Large-area ultrathin films of reduced graphene oxide as a transparent and flexible electronic material. Nat Nanotechnol. May 2008;3(5):270-274.

8. Compton OC, Nguyen ST. Graphene oxide, highly reduced graphene oxide, and graphene: versatile building blocks for carbon-based materials. Small. Mar 22 2010;6(6):711-723. 
9. Ritter KA, Lyding JW. The influence of edge structure on the electronic properties of graphene quantum dots and nanoribbons. Nat Mater. Mar 2009;8(3):235-242.

10. Pan D, Zhang J, Li Z, Wu M. Hydrothermal route for cutting graphene sheets into blueluminescent graphene quantum dots. Adv Mater. Feb 9 2010;22(6):734-738.

11. Peng J, Gao W, Gupta BK, et al. Graphene quantum dots derived from carbon fibers. Nano Lett. Feb 8 2012;12(2):844-849.

12. Yoo JM, Kang JH, Hong BH. Graphene-based nanomaterials for versatile imaging studies. Chem Soc Rev. Jul 21 2015;44(14):4835-4852.

13. Yang K, Feng L, Shi X, Liu Z. Nano-graphene in biomedicine: theranostic applications. Chem Soc Rev. Jan 21 2013;42(2):530-547.

14. Tang L, Wang Y, Li J. The graphene/nucleic acid nanobiointerface. Chem Soc Rev. Oct 7 2015;44(19):6954-6980.

15. Chen D, Tang L, Li J. Graphene-based materials in electrochemistry. Chem Soc Rev. Aug 2010;39(8):3157-3180.

16. Tang L, Wang Y, Liu Y, Li J. DNA-directed self-assembly of graphene oxide with applications to ultrasensitive oligonucleotide assay. ACS nano. May 24 2011;5(5):3817-3822.

17. Huang $\mathrm{P}, \mathrm{Xu} \mathrm{C}$, Lin J, et al. Folic acid-conjugated graphene oxide loaded with photosensitizers for targeting photodynamic therapy. Theranostics. 2011;1:240-250.

18. Zhang J, Yang H, Shen G, Cheng P, Zhang J, Guo S. Reduction of graphene oxide via L-ascorbic acid. Chem Commun (Camb). Feb 21 2010;46(7):1112-1114. 
19. Goenka S, Sant V, Sant S. Graphene-based nanomaterials for drug delivery and tissue engineering. J Control Release. Jan 10 2014;173:75-88.

20. Zhang J, Zhang F, Yang H, et al. Graphene oxide as a matrix for enzyme immobilization. Langmuir. 2010;26(9):6083-6085.

21. Haubner K, Murawski J, Olk P, et al. The route to functional graphene oxide. Chem Phys Chem. Jul 12 2010;11(10):2131-2139.

22. Kim YK, Kim MH, Min DH. Biocompatible reduced graphene oxide prepared by using dextran as a multifunctional reducing agent. Chem Commun (Camb). Mar 21 2011;47(11):3195-3197.

23. Li L, Wu G, Yang G, Peng J, Zhao J, Zhu JJ. Focusing on luminescent graphene quantum dots: current status and future perspectives. Nanoscale. May 21 2013;5(10):4015-4039.

24. Janib SM, Moses AS, MacKay JA. Imaging and drug delivery using theranostic nanoparticles. Adv Drug Deliver Rev. 2010;62(11):1052-1063.

25. Nguyen KT, Zhao Y. Integrated graphene/nanoparticle hybrids for biological and electronic applications. Nanoscale. Jun 21 2014;6(12):6245-6266.

26. Zhang H, Grüner G, Zhao Y. Recent advancements of graphene in biomedicine. J Mater Chem $B$. 2013;1(20):2542-2567.

27. Hummers Jr WS, Offeman RE. Preparation of graphitic oxide. J Am Chem Soc. 1958;80(6):13391339.

28. Shen J, Zhu Y, Yang X, Li C. Graphene quantum dots: emergent nanolights for bioimaging, sensors, catalysis and photovoltaic devices. Chem Commun. Apr 18 2012;48(31):3686-3699. 
29. Zhu S, Zhang J, Qiao C, et al. Strongly green-photoluminescent graphene quantum dots for bioimaging applications. Chem Commun. Jun 28 2011;47(24):6858-6860.

30. Sun H, Cao L, Lu L. Magnetite/reduced graphene oxide nanocomposites: one step solvothermal synthesis and use as a novel platform for removal of dye pollutants. Nano Res. 2011;4(6):550-562.

31. Zhou X, Huang X, Qi X, et al. In situ synthesis of metal nanoparticles on single-layer graphene oxide and reduced graphene oxide surfaces. J Phys Chem C. 2009;113(25):10842-10846.

32. Wang $X$, Huang $P$, Feng $L$, et al. Green controllable synthesis of silver nanomaterials on graphene oxide sheets via spontaneous reduction. RSC Adv. 2012;2(9):3816-3822.

33. Yang $\mathrm{X}$, Zhang $\mathrm{X}, \mathrm{Ma} \mathrm{Y}$, Huang $\mathrm{Y}$, Wang $\mathrm{Y}$, Chen $\mathrm{Y}$. Superparamagnetic graphene oxide- $\mathrm{Fe}_{3} \mathrm{O}_{4}$ nanoparticles hybrid for controlled targeted drug carriers. J Mater Chem. 2009;19(18):2710-2714.

34. Yang $X$, Wang $Y$, Huang $X$, et al. Multi-functionalized graphene oxide based anticancer drugcarrier with dual-targeting function and pH-sensitivity. J Mater Chem. 2011;21(10):3448-3454.

35. Yang K, Hu L, Ma X, et al. Multimodal imaging guided photothermal therapy using functionalized graphene nanosheets anchored with magnetic nanoparticles. Adv Mater. Apr 10 2012;24(14):1868-1872.

36. Huang J, Zhang L, Chen B, et al. Nanocomposites of size-controlled gold nanoparticles and graphene oxide: formation and applications in SERS and catalysis. Nanoscale. Dec 2010;2(12):2733-2738.

37. Badhulika S, Terse-Thakoor T, Villarreal C, Mulchandani A. Graphene hybrids: synthesis strategies and applications in sensors and sensitized solar cells. Front Chem. 2015;3:38.

38. Hu SH, Chen YW, Hung WT, Chen IW, Chen SY. Quantum-dot-tagged reduced graphene oxide nanocomposites for bright fluorescence bioimaging and photothermal therapy monitored in situ. Adv Mater. Apr 3 2012;24(13):1748-1754. 
39. Lin X, Xie J, Zhu L, et al. Hybrid ferritin nanoparticles as activatable probes for tumor imaging. Angew Chem Int Ed Engl. Feb 11 2011;50(7):1569-1572.

40. Wang C, Li J, Amatore C, Chen Y, Jiang H, Wang XM. Gold nanoclusters and graphene nanocomposites for drug delivery and imaging of cancer cells. Angew Chem Int Ed Engl. Dec 2 2011;50(49):11644-11648.

41. Nie L, Huang P, Li W, et al. Early-stage imaging of nanocarrier-enhanced chemotherapy response in living subjects by scalable photoacoustic microscopy. ACS nano. Dec 23 2014;8(12):12141-12150.

42. Liu Z, Robinson JT, Sun X, Dai H. PEGylated nanographene oxide for delivery of water-insoluble cancer drugs. J Am Chem Soc. Aug 20 2008;130(33):10876-10877.

43. Yan X, Niu G, Lin J, et al. Enhanced fluorescence imaging guided photodynamic therapy of sinoporphyrin sodium loaded graphene oxide. Biomaterials. Feb 2015;42:94-102.

44. Yang K, Zhang S, Zhang G, Sun X, Lee ST, Liu Z. Graphene in mice: ultrahigh in vivo tumor uptake and efficient photothermal therapy. Nano Lett. Sep 8 2010;10(9):3318-3323.

45. Robinson JT, Tabakman SM, Liang $\mathrm{Y}$, et al. Ultrasmall reduced graphene oxide with high nearinfrared absorbance for photothermal therapy. J Am Chem Soc. May 4 2011;133(17):6825-6831.

46. Sun Z, Huang $P$, Tong $G$, et al. VEGF-loaded graphene oxide as theranostics for multi-modality imaging-monitored targeting therapeutic angiogenesis of ischemic muscle. Nanoscale. 2013;5(15):68576866.

47. Peng C, Hu W, Zhou Y, Fan C, Huang Q. Intracellular imaging with a graphene-based fluorescent probe. Small. Aug 2 2010;6(15):1686-1692. 
48. Wang $Y$, Wang H, Liu D, Song S, Wang X, Zhang H. Graphene oxide covalently grafted upconversion nanoparticles for combined NIR mediated imaging and photothermal/photodynamic cancer therapy. Biomaterials. Oct 2013;34(31):7715-7724.

49. Huang $P$, Wang $S$, Wang $X$, et al. Surface functionalization of chemically reduced graphene oxide for targeted photodynamic therapy. J Biomed Nanotechnol. Jan 2015;11(1):117-125.

50. Guo C, Book-Newell B, Irudayaraj J. Protein-directed reduction of graphene oxide and intracellular imaging. Chem Commun (Camb). Dec 21 2011;47(47):12658-12660.

51. Li JL, Bao HC, Hou XL, Sun L, Wang XG, Gu M. Graphene oxide nanoparticles as a nonbleaching optical probe for two - photon luminescence imaging and cell therapy. Angew Chem Int Ed. 2012;51(8):1830-1834.

52. Wu H, Shi $\mathrm{H}$, Wang $\mathrm{Y}$, et al. Hyaluronic acid conjugated graphene oxide for targeted drug delivery. Carbon. 2014;69:379-389.

53. Konkena B, Vasudevan S. Covalently linked, water-dispersible, cyclodextrin: reduced-graphene oxide sheets. Langmuir. 2012;28(34):12432-12437.

54. Hong $\mathrm{H}$, Yang $\mathrm{K}$, Zhang $\mathrm{Y}$, et al. In vivo targeting and imaging of tumor vasculature with radiolabeled, antibody-conjugated nanographene. ACS nano. Mar 27 2012;6(3):2361-2370.

55. Zhang Y, Nayak TR, Hong H, Cai W. Graphene: a versatile nanoplatform for biomedical applications. Nanoscale. Jul 7 2012;4(13):3833-3842.

56. Hong $\mathrm{H}$, Zhang $\mathrm{Y}$, Engle JW, et al. In vivo targeting and positron emission tomography imaging of tumor vasculature with ${ }^{66} \mathrm{Ga}$-labeled nano-graphene. Biomaterials. Jun 2012;33(16):4147-4156.

57. Fazaeli $\mathrm{Y}$, Akhavan O, Rahighi R, Aboudzadeh MR, Karimi E, Afarideh H. In vivo SPECT imaging of tumors by ${ }^{198,199}$ Au-labeled graphene oxide nanostructures. Mater Sci Eng C. Dec 2014;45:196-204. 
58. Cornelissen B, Able S, Kersemans V, et al. Nanographene oxide-based radioimmunoconstructs for in vivo targeting and SPECT imaging of HER2-positive tumors. Biomaterials. Jan 2013;34(4):11461154.

59. Yang K, Gong H, Shi X, Wan J, Zhang Y, Liu Z. In vivo biodistribution and toxicology of functionalized nano-graphene oxide in mice after oral and intraperitoneal administration. Biomaterials. Apr 2013;34(11):2787-2795.

60. Yang $\mathrm{K}$, Wan J, Zhang $\mathrm{S}$, Zhang $\mathrm{Y}$, Lee $\mathrm{ST}$, Liu Z. In vivo pharmacokinetics, long-term biodistribution, and toxicology of PEGylated graphene in mice. ACS nano. Jan 25 2011;5(1):516-522.

61. Wang J, Mi P, Lin G, Wang YX, Liu G, Chen X. Imaging-guided delivery of RNAi for anticancer treatment. Adv Drug Deliv Rev. Jan 222016.

62. Debbage $\mathrm{P}$, Jaschke $\mathrm{W}$. Molecular imaging with nanoparticles: giant roles for dwarf actors. Histochem Cell Biol. Nov 2008;130(5):845-875.

63. Park K, Lee S, Kang E, Kim K, Choi K, Kwon IC. New generation of multifunctional nanoparticles for cancer imaging and therapy. Adv Funct Mater. 2009;19(10):1553-1566.

64. Smith AM, Mancini MC, Nie S. Bioimaging: second window for in vivo imaging. Nat Nanotechnol. Nov 2009;4(11):710-711.

65. Hong G, Zou Y, Antaris AL, et al. Ultrafast fluorescence imaging in vivo with conjugated polymer fluorophores in the second near-infrared window. Nat Commun. 2014;5:4206.

66. Robinson JT, Hong G, Liang Y, Zhang B, Yaghi OK, Dai H. In vivo fluorescence imaging in the second near-infrared window with long circulating carbon nanotubes capable of ultrahigh tumor uptake. J Am Chem Soc. Jun 27 2012;134(25):10664-10669. 
67. Liu Q, Guo B, Rao Z, Zhang B, Gong JR. Strong two-photon-induced fluorescence from photostable, biocompatible nitrogen-doped graphene quantum dots for cellular and deep-tissue imaging. Nano Lett. Jun 12 2013;13(6):2436-2441.

68. Chung C, Kim YK, Shin D, Ryoo SR, Hong BH, Min DH. Biomedical applications of graphene and graphene oxide. Acc Chem Res. Oct 15 2013;46(10):2211-2224.

69. Baker SN, Baker GA. Luminescent carbon nanodots: emergent nanolights. Angew Chem Int Ed. Sep 10 2010;49(38):6726-6744.

70. Zhu S, Meng Q, Wang L, et al. Highly photoluminescent carbon dots for multicolor patterning, sensors, and bioimaging. Angew Chem Int Ed. Apr 2 2013;52(14):3953-3957.

71. Sun X, Liu Z, Welsher K, et al. Nano-graphene oxide for cellular imaging and drug delivery. Nano research. 2008;1(3):203-212.

72. Rong $\mathrm{P}$, Yang $\mathrm{K}$, Srivastan $\mathrm{A}$, et al. Photosensitizer loaded nano-graphene for multimodality imaging guided tumor photodynamic therapy. Theranostics. 2014;4(3):229-239.

73. Abdullah $\mathrm{Al} \mathrm{N}$, Lee JE, In I, et al. Target delivery and cell imaging using hyaluronic acidfunctionalized graphene quantum dots. Mol Pharm. Oct 7 2013;10(10):3736-3744.

74. Ge J, Lan M, Zhou B, et al. A graphene quantum dot photodynamic therapy agent with high singlet oxygen generation. Nat Commun. 2014;5:4596.

75. Liu Z, Guo Z, Zhong H, Qin X, Wan M, Yang B. Graphene oxide based surface-enhanced Raman scattering probes for cancer cell imaging. Phys Chem Chem Phys. Feb 28 2013;15(8):2961-2966.

76. Liu Q, Wei L, Wang J, et al. Cell imaging by graphene oxide based on surface enhanced Raman scattering. Nanoscale. Nov 21 2012;4(22):7084-7089. 
77. Kim YK, Han SW, Min DH. Graphene oxide sheath on Ag nanoparticle/graphene hybrid films as an antioxidative coating and enhancer of surface-enhanced Raman scattering. ACS Appl Mater Interfaces. Dec 2012;4(12):6545-6551.

78. Ma X, Qu Q, Zhao $Y$, et al. Graphene oxide wrapped gold nanoparticles for intracellular Raman imaging and drug delivery. J Mater Chem B. 2013;1(47):6495-6500.

79. Wang Y, Polavarapu L, Liz-Marzan LM. Reduced graphene oxide-supported gold nanostars for improved SERS sensing and drug delivery. ACS Appl Mater Interfaces. Dec 24 2014;6(24):21798-21805.

80. Huang J, Zong C, Shen $\mathrm{H}$, et al. Mechanism of cellular uptake of graphene oxide studied by surface-enhanced Raman spectroscopy. Small. Aug 20 2012;8(16):2577-2584.

81. Song ZL, Chen Z, Bian X, et al. Alkyne-functionalized superstable graphitic silver nanoparticles for Raman imaging. J Am Chem Soc. Oct 1 2014;136(39):13558-13561.

82. $\mathrm{Hu} \mathrm{YH}$. The first magnetic-nanoparticle-free carbon-based contrast agent of magnetic-resonance imaging-fluorinated graphene oxide. Small. Apr 24 2014;10(8):1451-1452.

83. Strijkers GJ, Mulder WJ, van Tilborg GA, Nicolay K. MRI contrast agents: current status and future perspectives. Anti-Cancer Agents Med Chem. May 2007;7(3):291-305.

84. Caravan P, Ellison JJ, McMurry TJ, Lauffer RB. Gadolinium(III) chelates as MRI contrast agents: structure, dynamics, and applications. Chem Rev. Sep 8 1999;99(9):2293-2352.

85. Gizzatov A, Keshishian V, Guven A, et al. Enhanced MRI relaxivity of aquated $\mathrm{Gd}^{3+}$ ions by carboxyphenylated water-dispersed graphene nanoribbons. Nanoscale. Mar 21 2014;6(6):3059-3063.

86. Yang HW, Huang $\mathrm{CY}$, Lin $\mathrm{CW}$, et al. Gadolinium-functionalized nanographene oxide for combined drug and microRNA delivery and magnetic resonance imaging. Biomaterials. Aug 2014;35(24):6534-6542. 
87. Chen W, Yi P, Zhang Y, Zhang L, Deng Z, Zhang Z. Composites of aminodextran-coated $\mathrm{Fe}_{3} \mathrm{O}_{4}$ nanoparticles and graphene oxide for cellular magnetic resonance imaging. ACS Appl Mater Interfaces. Oct 2011;3(10):4085-4091.

88. F127 EoaP, functionalized magnetite/grapheneWang S, Zhang Q, et al. Magnetic graphenebased nanotheranostic agent for dual-modality mapping guided photothermal therapy in regional lymph nodal metastasis of pancreatic cancer. Biomaterials. Nov 2014;35(35):9473-9483.

89. Huang $\mathrm{P}$, Rong $\mathrm{P}$, Lin J, et al. Triphase interface synthesis of plasmonic gold bellflowers as nearinfrared light mediated acoustic and thermal theranostics. J Am Chem Soc. Jun 11 2014;136(23):83078313.

90. Nie L, Chen X. Structural and functional photoacoustic molecular tomography aided by emerging contrast agents. Chem Soc Rev. 2014;43(20):7132-7170.

91. Lalwani G, Cai X, Nie L, Wang LV, Sitharaman B. Graphene-based contrast agents for photoacoustic and thermoacoustic tomography. Photoacoustics. Dec 1 2013;1(3-4):62-67.

92. Patel MA, Yang $\mathrm{H}$, Chiu PL, et al. Direct production of graphene nanosheets for near infrared photoacoustic imaging. ACS nano. Sep 24 2013;7(9):8147-8157.

93. Wang $\mathrm{Y}-\mathrm{W}, \mathrm{Fu} \mathrm{Y}-\mathrm{Y}$, Peng $\mathrm{Q}$, et al. Dye-enhanced graphene oxide for photothermal therapy and photoacoustic imaging. J Mater Chem B. 2013;1(42):5762-5767.

94. Sheng Z, Song L, Zheng J, et al. Protein-assisted fabrication of nano-reduced graphene oxide for combined in vivo photoacoustic imaging and photothermal therapy. Biomaterials. Jul 2013;34(21):52365243.

95. Lusic H, Grinstaff MW. X-ray-computed tomography contrast agents. Chem Rev. Mar 13 2013;113(3):1641-1666. 
96. Lee N, Choi SH, Hyeon T. Nano-sized CT contrast agents. Adv Mater. May 21 2013;25(19):26412660.

97. Shi J, Wang L, Zhang J, et al. A tumor-targeting near-infrared laser-triggered drug delivery system based on GO@Ag nanoparticles for chemo-photothermal therapy and X-ray imaging. Biomaterials. Jul 2014;35(22):5847-5861.

98. Zhang $\mathrm{H}, \mathrm{Wu} \mathrm{H}$, Wang J, et al. Graphene oxide-BaGdF 5 nanocomposites for multi-modal imaging and photothermal therapy. Biomaterials. Feb 2015;42:66-77.

99. Shi X, Gong H, Li Y, Wang C, Cheng L, Liu Z. Graphene-based magnetic plasmonic nanocomposite for dual bioimaging and photothermal therapy. Biomaterials. Jul 2013;34(20):4786-4793.

100. Jin Y, Wang J, Ke H, Wang S, Dai Z. Graphene oxide modified PLA microcapsules containing gold nanoparticles for ultrasonic/CT bimodal imaging guided photothermal tumor therapy. Biomaterials. Jul 2013;34(20):4794-4802.

101. Huang $\mathrm{P}$, Rong $\mathrm{P}$, Jin $\mathrm{A}$, et al. Dye-loaded ferritin nanocages for multimodal imaging and photothermal therapy. Adv Mater. Oct 8 2014;26(37):6401-6408.

102. Ma J, Huang $\mathrm{P}, \mathrm{He} \mathrm{M}$, et al. Folic acid-conjugated $\mathrm{LaF}_{3}: \mathrm{Yb}, \mathrm{Tm} @ \mathrm{SiO}_{2}$ nanoprobes for targeting dual-modality imaging of upconversion luminescence and X-ray computed tomography. J Phys Chem B. Dec 6 2012;116(48):14062-14070.

103. Cheng Z, Al Zaki A, Hui JZ, Muzykantov VR, Tsourkas A. Multifunctional nanoparticles: cost versus benefit of adding targeting and imaging capabilities. Science. Nov 16 2012;338(6109):903-910.

104. Yan $\mathrm{X}, \mathrm{Hu} \mathrm{H}$, Lin J, et al. Optical and photoacoustic dual-modality imaging guided synergistic photodynamic/photothermal therapies. Nanoscale. Feb 14 2015;7(6):2520-2526. 
105. Yang K, Feng L, Liu Z. The advancing uses of nano-graphene in drug delivery. Expert Opin Drug Deliv. Apr 2015;12(4):601-612.

106. Liu J, Cui L, Losic D. Graphene and graphene oxide as new nanocarriers for drug delivery applications. Acta Biomater. 2013;9(12):9243-9257.

107. Liu Q, Shi J, Sun J, Wang T, Zeng L, Jiang G. Graphene and graphene oxide sheets supported on silica as versatile and high-performance adsorbents for solid-phase extraction. Angew Chem Int Ed Engl. Jun 20 2011;50(26):5913-5917.

108. Song J, Yang X, Jacobson $\mathrm{O}$, et al. Sequential drug release and enhanced photothermal and photoacoustic effect of hybrid reduced graphene oxide-loaded ultrasmall gold nanorod vesicles for cancer therapy. ACS nano. Sep 22 2015;9(9):9199-9209.

109. Ryoo SR, Lee J, Yeo J, et al. Quantitative and multiplexed microRNA sensing in living cells based on peptide nucleic acid and nano graphene oxide (PANGO). ACS nano. Jul 23 2013;7(7):5882-5891.

110. Wang Y, Li Z, Hu D, Lin CT, Li J, Lin Y. Aptamer/graphene oxide nanocomplex for in situ molecular probing in living cells. J Am Chem Soc. Jul 14 2010;132(27):9274-9276.

111. Wang $Z$, Huang $P$, Bhirde A, et al. A nanoscale graphene oxide-peptide biosensor for real-time specific biomarker detection on the cell surface. Chem Commun (Camb). Oct 9 2012;48(78):9768-9770.

112. Tang $\mathrm{Y}, \mathrm{Hu} \mathrm{H}$, Zhang MG, et al. An aptamer-targeting photoresponsive drug delivery system using "off-on" graphene oxide wrapped mesoporous silica nanoparticles. Nanoscale. Apr 14 2015;7(14):6304-6310.

113. Sreejith S, Ma X, Zhao Y. Graphene oxide wrapping on squaraine-loaded mesoporous silica nanoparticles for bioimaging. J Am Chem Soc. Oct 24 2012;134(42):17346-17349. 
114. Raccichini R, Varzi A, Passerini S, Scrosati B. The role of graphene for electrochemical energy storage. Nat Mater. Mar 2015;14(3):271-279.

115. Jiang L, Fan Z. Design of advanced porous graphene materials: from graphene nanomesh to 3D architectures. Nanoscale. Feb 21 2014;6(4):1922-1945.

116. Wang $\mathrm{Y}$, Wang $\mathrm{K}$, Zhao J, et al. Multifunctional mesoporous silica-coated graphene nanosheet used for chemo-photothermal synergistic targeted therapy of glioma. J Am Chem Soc. Mar 27 2013;135(12):4799-4804.

117. Cong HP, Chen JF, Yu SH. Graphene-based macroscopic assemblies and architectures: an emerging material system. Chem Soc Rev. Nov 7 2014;43(21):7295-7325.

118. Zhu Y, Murali S, Cai W, et al. Graphene and graphene oxide: synthesis, properties, and applications. Adv Mater. Sep 15 2010;22(35):3906-3924.

119. Morales Narváez E, Merkoçi A. Graphene oxide as an optical biosensing platform. Adv Mater. 2012;24(25):3298-3308.

120. Chien CT, Li SS, Lai WJ, et al. Tunable photoluminescence from graphene oxide. Angew Chem Int Ed. Jul 2 2012;51(27):6662-6666.

121. Cote $L$, Cruz-Silva R, Huang J. Flash reduction and patterning of graphite oxide and its polymer composite. J Am Chem Soc. Aug 12 2009;131(31):11027-11032.

122. Gong H, Peng R, Liu Z. Carbon nanotubes for biomedical imaging: the recent advances. Adv Drug Deliv Rev. Dec 2013;65(15):1951-1963.

123. Hong G, Lee JC, Robinson JT, et al. Multifunctional in vivo vascular imaging using near-infrared II fluorescence. Nat Med. 2012;18(12):1841-1846. 
124. Hong G, Diao S, Chang J, et al. Through-skull fluorescence imaging of the brain in a new nearinfrared window. Nat Photonics. 2014;8(9):723-730.

125. Ferrari AC, Basko DM. Raman spectroscopy as a versatile tool for studying the properties of graphene. Nat Nanotechnol. 2013;8(4):235-246.

126. Malard L, Pimenta M, Dresselhaus G, Dresselhaus M. Raman spectroscopy in graphene. Phys Rep. 2009;473(5):51-87.

127. Rao AM, Richter E, Bandow S, et al. Diameter-selective Raman scattering from vibrational modes in carbon nanotubes. Science. 1997;275(5297):187-191.

128. Robinson JT, Tabakman SM, Liang $\mathrm{Y}$, et al. Ultrasmall reduced graphene oxide with high nearinfrared absorbance for photothermal therapy. J Am Chem Soc. 2011;133(17):6825-6831.

129. Wang $\mathrm{K}$, Ruan J, Song $\mathrm{H}$, et al. Biocompatibility of graphene oxide. Nanoscale Res Lett. 2011;6(8):1. 